Direct evaluation of radiobiological parameters from clinical data in the case of ion beam therapy: an alternative approach to the relative biological effectiveness

This content has been downloaded from IOPscience. Please scroll down to see the full text. 2014 Phys. Med. Biol. 597393

(http://iopscience.iop.org/0031-9155/59/23/7393)

View the table of contents for this issue, or go to the journal homepage for more

Download details:

IP Address: 137.138.139.20

This content was downloaded on 12/11/2014 at 09:18

Please note that terms and conditions apply. 


\title{
Direct evaluation of radiobiological parameters from clinical data in the case of ion beam therapy: an alternative approach to the relative biological effectiveness
}

\author{
A Cometto ${ }^{1}$, G Russo ${ }^{2}$, F Bourhaleb ${ }^{3}, \mathrm{~F} \mathrm{M} \mathrm{Milian}^{4}$, \\ S Giordanengo ${ }^{2}$, F Marchetto $^{2}, \mathbf{R}$ Cirio $^{2,5}$ and A Attili ${ }^{2}$ \\ 1 Politecnico di Torino, Corso Duca degli Abruzzi, 24, 10129 Torino, Italy \\ 2 Istituto Nazionale di Fisica Nucleare (INFN), Torino, Italy \\ ${ }^{3}$ I-See, Internet Simulation Evaluation Envision Ltd, Via P. Giuria, 110125 Turin \\ Torino, Italy \\ ${ }^{4}$ Universidade Estadual de Santa Cruz, Rodovia Jorge Amado, Km 16-Salobrinho, \\ Ilhéus 45662-900, Brazil \\ ${ }^{5}$ Università degli Studi di Torino, Via Giuseppe Verdi 8, Torino, Italy \\ E-mail: attili@to.infn.it
}

Received 26 June 2014, revised 15 September 2014

Accepted for publication 9 October 2014

Published 11 November 2014

\begin{abstract}
The relative biological effectiveness (RBE) concept is commonly used in treatment planning for ion beam therapy. Whether models based on in vitrol in vivo $\mathrm{RBE}$ data can be used to predict human response to treatments is an open issue. In this work an alternative method, based on an effective radiobiological parameterization directly derived from clinical data, is presented. The method has been applied to the analysis of prostate cancer trials with protons and carbon ions.

Prostate cancer trials with proton and carbon ion beams reporting 5 yearlocal control (LC5) and grade 2 (G2) or higher genitourinary toxicity rates (TOX) were selected from literature to test the method. Treatment simulations were performed on a representative subset of patients to produce dose and linear energy transfer distribution, which were used as explicative physical variables for the radiobiological modelling. Two models were taken into consideration: the microdosimetric kinetic model (MKM) and a linear model (LM). The radiobiological parameters of the LM and MKM were obtained by coupling them with the tumor control probability and normal tissue complication probability models to fit the LC5 and TOX data through likelihood maximization. The model ranking was based on the Akaike information criterion.
\end{abstract}


Results showed large confidence intervals due to the limited variety of available treatment schedules. $\mathrm{RBE}$ values, such as $\mathrm{RBE}=1.1$ for protons in the treated volume, were derived as a by-product of the method, showing a consistency with current approaches. Carbon ion RBE values were also derived, showing lower values than those assumed for the original treatment planning in the target region, whereas higher values were found in the bladder. Most importantly, this work shows the possibility to infer the radiobiological parametrization for proton and carbon ion treatment directly from clinical data.

Keywords: RBE, ion beam therapy, treatment planning

(Some figures may appear in colour only in the online journal)

\section{Introduction}

The adoption of ions into radiation therapy (RT) aims at improving the physical selectivity of the irradiation, characterized by a favorable depth-dose profile (the Bragg curve). In addition, heavy ions offer an even greater efficacy for tumor treatments thanks to the enhanced biological effect of their high linear energy transfer (LET). The knowledge of the radiobiology properties of ion beams, necessary in treatment planning systems (TPSs), is expressed through the evaluation of the relative biological effectiveness (RBE). The purpose of the RBE determination is to help in prescribing the appropriate photon-equivalent dose, here referred to as $\mathrm{RBE}$ weighted dose (RWD), $D_{\mathrm{RBE}}=D \times \mathrm{RBE}$, for which the radiation-oncologists have accumulated a wider clinical experience.

The complexity in measuring relevant biological effects to produce accurate mathematical models that link dose and LET spectra to clinical response remains one of the main problems. Amongst clinical centers, the RBE evaluation has been based mainly on in vitro and in vivo cell survival data. It is assumed that the RBE systematics found for in vitro and in vivo also remain true for clinical data, thus permitting the use of these estimates as surrogate of the 'clinical RBE' to predict the clinical treatment outcome. Following the linear-quadratic (LQ) formalism, the initial $\operatorname{RBE}\left(\alpha / \alpha_{X}\right)$ after ion beam irradiation mainly depends on the $\alpha_{X} / \beta_{X}$ ratio for the reference radiation (Koike et al 2002, Friedrich et al 2012b). The reduction of the required input information to the $\alpha_{X} / \beta_{X}$ ratios also increases the range of applicability, since this ratio is known for many tumor and normal tissues. These assumptions fill a knowledge gap between the in vitro/in vivo experimental and clinical RBE determinations.

Several different approaches are used in clinical settings to address the RBE evaluation of ion beams. In the case of proton beams, the RBE variation observed from in vivo studies is small enough to assume a constant value of 1.1 relative to a MV photon beam (Paganetti et al 2002) according to the ICRU Report 78 recommendations (ICRU 2007). Recent studies have suggested that the experimentally observed deviations from $\mathrm{RBE}=1.1$ (Petrović et al 2010, Calugaru et al 2011) could produce clinically discernible effects. These should be considered especially for single-field treatments and when organs at risk (OARs) are located immediately distal to the Bragg peak (Tilly et al 2005, Frese et al 2011, Grassberger et al 2011, Giantsoudi et al 2013, Grun et al 2013). However, the clinical relevance of these RBE variations is still a matter of debate (Sethi et al 2014).

For carbon ion-beam therapy with carbons the RWD has to be explicitly evaluated and optimized in the planning procedure (IAEA 2008). This is generally a difficult task due to the manifold dependencies of RBE (particle type, kinetic energy, fractionation scheme, dosedelivery time structure, cell or tissue type) and to the complex mixed radiation field in the 
patient during the irradiation. The few institutes using carbon ion beams for treatment have taken different approaches for calculating the effective dose. At Heavy Ion Medical Accelerator in Chiba (HIMAC) and Hyogo Ion Beam Medical Center (HIBMC) extensive beam-specific in vitro cell-survival measurements are used to model the RBE as a function of depth, LET and/or kinetic energy. The absolute dose level is determined by adjusting the RBE for carbon ions at the distal part of the spread out Bragg peak (SOBP) to coincide with the RBE used in neutron therapy (Kanai et al 1997, Kanai et al 1999, Kagawa et al 2002). In particular, the design of the SOBP at HIMAC is based on in vitro measurements of the human salivary gland (HSG) tumor cell line as end-point surrogate (Matsufuji et al 2007). Other approaches are based on the development and implementation of more comprehensive biologic models, such as the local effect model (LEM) (Scholz and Kraft 1996, Scholz et al 1997, Elsässer and Scholz 2007, Friedrich et al 2012a) and the microdosimetric-kinetic model (MKM) (Hawkins 1998, Hawkins 2003). Although the mechanics of the models are different, their basic assumptions are similar and they have been shown to produce similar output (Kase et al 2008). In particular, the European facilities, such as Heidelberg Ion-Beam Therapy Center (HIT) and Centro Nazionale di Adroterapia Oncologica in Pavia (CNAO), use the LEM with a parametrization corresponding to a unique representative cell line, with an $\alpha_{X} / \beta_{X}$ ratio fixed at $2 \mathrm{~Gy}$, derived from mice brain in vivo measurements (Krämer and Scholz 2000, Jäkel et al 2007, Fossati et al 2012). The LEM was also benchmarked in retrospective analysis to evaluate clinical dose-effect probabilities from the dose volume histograms (DVHs) of treated patients (Schlampp et al 2011).

Several issues can be easily identified within the present RBE-based approaches. Large uncertainties are involved in the currently in vitro RBE estimations, which were fitted to set of experimental cell survival data for protons and carbon ions. Inaccuracy in RBE estimation remains the main source of uncertainty in the prescribed dose, in particular for carbon ion therapy (Durante and Loeffler 2010, Jones et al 2011). These fluctuations are irreducible: the RBE values from in vitro experiment in the past 50 years show a high variance and this spread cannot be reduced by repeating similar experiments (Friedrich et al 2012b, Loeffler and Durante 2013). There is also the fundamental question about the RBE translatability from in vitro to in vivo conditions. For example, it is well known that RBE variations tend to be smaller for in vivo systems, in particular the dependence on dose, i.e. the increasing of RBE with decreasing dose, appears to be weaker in vivo compared to the in vitro data (Paganetti et al 2002). Moreover, the type of cell lines used in these in vitro/in vivo experiments may not be representative of the different types and stages of tumors and healthy tissues involved in treatments. In this regard the RBE value for late-reacting normal tissues and slow-growing tumors could be different (and higher) for acute-reacting normal tissues and rapidly growing tumors (Jones et al 2011). Furthermore, the reference biological parameterization used in photon radiotherapy (e.g. the LQ parameters $\alpha_{X}$ and $\beta_{X}$ ) has a large variance, introducing an additional layer of uncertainty in the RBE based approaches (Bentzen and Joiner 2009, Bentzen et al 2010, Deasy et al 2010, Jackson et al 2010).

The aim of this work is to present an alternative method to the standard RBE-based treatment planning approaches, depending on the evaluation of the absolute biological effect that can be linked more directly to the clinical outcome of ion beam treatments. We evaluated the biological effects at the cell level via specific radiobiological models in the framework of the standard LQ model. Tumor control probability (TCP) and normal tissue complication probability (NTCP) have been coupled to the radiobiological models and used to infer the optimal radiobiological parameters for each specific end-point from the clinical data. The LQ parameters obtained may vary inside the patient, depending on the complex anatomical geometry of the patient, the mixed-LET spatially-varying quality of the irradiation and the specific tissues/ 


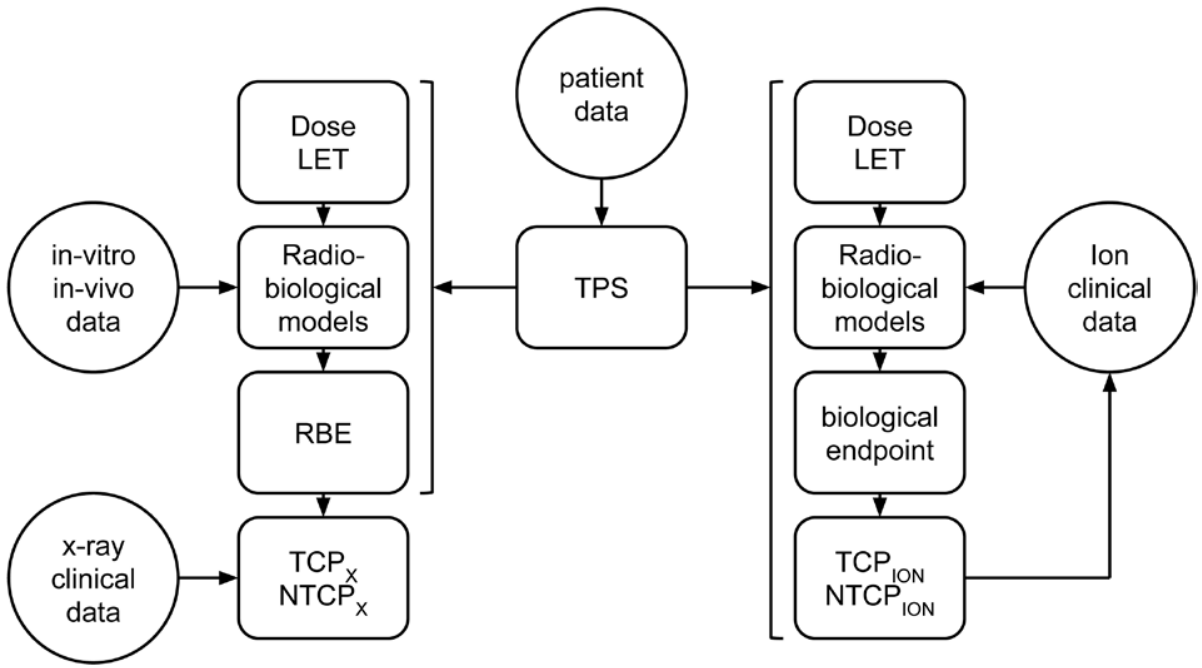

Figure 1. On the right-hand side, the flowchart outlining the proposed treatment planning procedure is shown. On the left-hand side the standard approach is displayed.

endpoints, affecting the RWD dose distribution. RBE distributions have been obtained as a byproduct of the general procedure to make comparisons with the current standard approaches reported in literature. The method has been tested for the treatment of prostate carcinoma with protons and carbon ions beam. More specifically, concerning the toxicity rates, late bladder complications were analyzed.

\section{Materials and methods}

\subsection{General workflow}

On the left side of the flowchart in figure 1, the standard RBE-based procedure commonly followed in treatment planning for ion beam therapy is shown while the workflow on the right summarizes the alternative procedure proposed in this work. In the first step the absorbed dose $D$ and LET distributions were computed based on representative patients and treatment definitions. The estimation of the biological and clinical effects was performed in the following two steps, namely the 'radiobiological' evaluation, i.e. the evaluation of the probability to observe a specific absolute effect at the cell level (such as cell survival of tumor or healthy tissues) and TCP and NTCP evaluations. By comparing the predicted TCP and NTCP with the observed clinical outcomes in terms of patient survival (or local control, or biochemical relapse) and toxicity, it was possible, through a fitting procedure, to infer the optimal effective parametrization of the biological and clinical models for ion treatments. These steps are detailed in the following sections.

\subsection{Clinical data and treatment definition}

To illustrate and test the method, an exemplifying application using a series of published clinical datasets describing the dose response of patients treated with proton and carbon ion beams was performed. For this purpose, a literature search using PubMED, aimed at identifying 
Table 1. Clinical data of prostate cancer trials with proton and carbon ion irradiations. In the case of protons, two laterally opposed fields were used, while for carbon ions one anterior-posterior port and a pair of lateral ports were used. $N_{f}$ and $N_{p}$ are the number of fractions and the number of patients, respectively.

\begin{tabular}{llllllllll}
\hline Reference & Ion & $D_{f}[\mathrm{GyE}]$ & $D_{\text {tot }}[\mathrm{GyE}]$ & $N_{f}$ & RBE & Beam setup & $N_{p}$ & bRFS & TOX \\
\hline Mayahara2007 & ${ }^{1} \mathrm{H}$ & 2 & 74 & 37 & 1.1 & 2 fields & 287 & $81 \%(5 \mathrm{yr})$ & $1 \% \mathrm{G} 3$ \\
Okada2012 & ${ }^{12} \mathrm{C}$ & 3.15 & 63 & 20 & $3^{\mathrm{a}}$ & 3 fields & 216 & $90.2 \%(5 \mathrm{yr})$ & $6.5 \% \mathrm{G} 2$ \\
Okada2012 & ${ }^{12} \mathrm{C}$ & 3.6 & 57.6 & 16 & $3^{\mathrm{a}}$ & 3 fields & 198 & $88.5 \%(5 \mathrm{yr})$ & $2 \% \mathrm{G} 2$ \\
\hline
\end{tabular}

a In distal SOBP.

relevant published prostate cancer trials with ion beams, was carried out. Only papers providing the necessary details to implement the treatment simulations, such as delivery type, treatment schedule, beam setup, optimization method, RBE and number of patients, were considered. The designed papers report clinical trials performed in HIMAC and HIBMC (table 1) (Mayahara et al 2007, Okada et al 2012). The selected data consist of three groups of patients identified by their treatment protocols, one with proton and two with carbon ion beams, the latter corresponding to two different fractionation schemes. As surrogate of TCP we focused on the 5 year biochemical relapse-free survival (bRFS), while for the NTCP we considered G2 or higher bladder (genitourinary) toxicity (TOX).

\subsection{Patient model and reference treatment planning}

The proposed evaluation of the TCP/NTCP relies on the knowledge of the dose and $\mathrm{LET}_{d}$ distributions of the individual patients. Since individual patient data were not available for this study, an approximation was introduced using a representative patient set. Computed tomography (CT) data from six representative prostate cancer patients and corresponding contoured structures were obtained from a clinical database and used. The six patients were assumed to be representative of the inter-patient anatomical variability that could be expected for a generic class of patients. Following the latter premise, the same six patients CT and structure data sets were used to evaluate the dose and $\mathrm{LET}_{d}$ distributions for each group (defined in the previous section), using the corresponding irradiation setups reported in table 1. This assumption is in particular acceptable for the purpose of the paper, the primary goal of which is to demonstrate the feasibility of the method and to illustrate an exemplary application.

The reported biological dose prescriptions and beam setup were used to implement the treatment planning simulations in which patients were irradiated with proton with $D_{f}=2 \mathrm{GyE}$ dose per fraction (Mayahara et al 2007) and with carbon ions with $D_{f}=3.15$ and 3.6 GyE dose per fraction (Okada et al 2012). In the case of the prostate, the standard technique for proton treatments consisted of two lateral opposed fields, while for carbon ion, three beams were used (2 lateral and one anterior-posterior). The TPS PlanKIT, developed and validated by INFN/IBA for proton and carbon ion beam therapy (Russo et al 2014), was used to optimize the treatment plans and to evaluate the putative dose and dose averaged LET $\left(\mathrm{LET}_{d}\right)$ distributions in the patients. In the planning simulations a computing grid voxel size of $3 \times 3 \times 3 \mathrm{~mm}^{3}$ was used.

Concerning the proton treatments, plans were optimized assuming a constant $\mathrm{RBE}=1.1$ in the whole patient (Mayahara et al 2007). In the case of carbon ions, the plans were biologically optimized using the MKM. The reference MKM parametrization was selected in order to reproduce in vitro cell survival data extracted from the Particle Irradiation Data Ensemble 

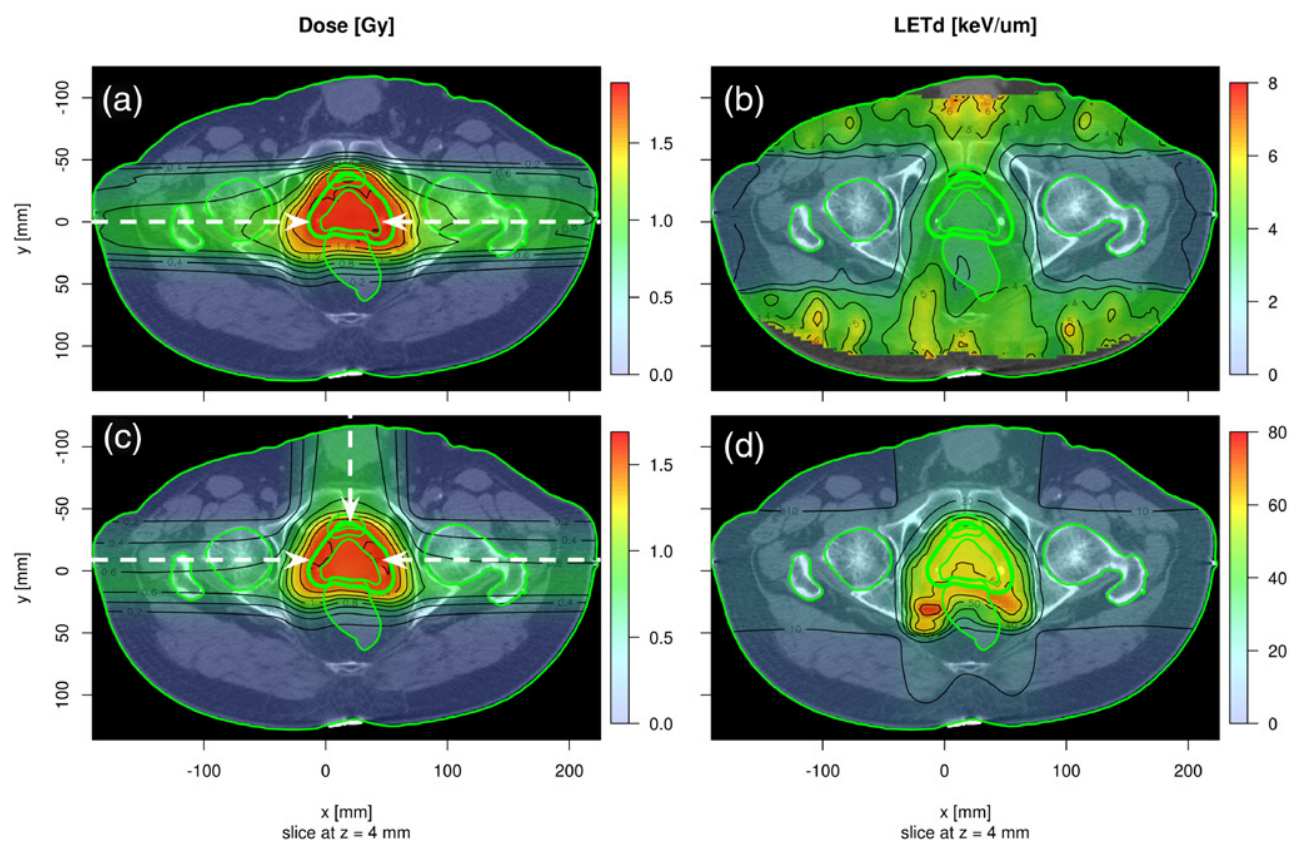

Figure 2. Example of dose (panels $(a)$ and $(c)$ ) and LET Lanels $_{d}(b)$ and $(d)$ ) distributions evaluated using the PlanKIT TPS for one patient irradiated with proton with $D_{f}=2 \mathrm{GyE}$ dose per fraction (panels $(a)$ and $(b)$ ) and carbon ion beams with $D_{f}=3.6 \mathrm{GyE}$ (panels $(c)$ and $(d)$ ). The white arrows indicate the main beam-ports and their directions.

(PIDE) database provided by the Gesellschaft für Schwerionenforschung (GSI) (Friedrich et al 2012b) and corresponding to a hypothetical tissue type with ratio $R=\alpha_{X} / \beta_{X}=3$ Gy for late responding tissues. We verified that with this parametrization we were able to reproduce a $\mathrm{RBE}=3$ in the distal part of the SOBP as reported in Okada et al (2012). The planning target volume (PTV) tolerance margins were adjusted until isodoses and PTV/bladder DVHs were similar to those reported in Mayahara et al (2007) and Okada et al (2012). The treatment planning evaluations described in this section are identified in this work as 'reference plans'. An example of the obtained dose and $\mathrm{LET}_{d}$ distributions of a treatment is shown in figure 2.

\subsection{Radiobiological modelling}

The first step in biological modelling, leading to the evaluation of the probability of specific biological endpoints at the voxel level, is outlined in the context of the LQ model:

$$
p\left(D, \mathrm{LET}_{d}\right)=\exp \left(-\alpha\left(\mathrm{LET}_{d}\right) D-\beta\left(\mathrm{LET}_{d}\right) D^{2}\right)
$$

where $\mathrm{LET}_{d}$, evaluated by the TPS, represents the dose-averaged LET in a specific voxel and was used as a surrogate for spectral characteristics of the energy deposition.

Regardless of the complex dependence between RBE and LET, it has been demonstrated that the $\mathrm{LET}_{d}$, together with the dose, can be effectively used to completely characterize the biological effect of the irradiation. In fact, several in vitro and in vivo studies have proven that, for a given endpoint, the proton RBE values show a linear dependence on LET in the dose and energy ranges clinically adopted (Paganetti et al 2002, Carabe et al 2012). A simple 
Table 2. Values of the parameters used for the reference $x$-ray radiation (Mavroidis et al 2013). Note: $D_{50}$ is the $50 \%$ response dose, $\gamma$ is the maximum normalized slope of the dose-response curve and $s$ is the relative seriality parameter.

\begin{tabular}{lllllll}
\hline Type & $D_{50}[\mathrm{~Gy}]$ & $\gamma$ & $\frac{\alpha_{X}}{\beta_{X}}[\mathrm{~Gy}]$ & $\alpha_{X}\left[\mathrm{~Gy}^{-1}\right]$ & $\beta_{X}\left[\mathrm{~Gy}^{-2}\right]$ & $s$ \\
\hline CTV(prostate) & 62 & 4 & 2 & 0.0906 & 0.0453 & - \\
Bladder & 80 & 3 & 3 & 0.0639 & 0.0213 & 0.18 \\
\hline
\end{tabular}

relationship was also used for proton in the range between $1-20 \mathrm{keV} \mu \mathrm{m}^{-1}$ to perform treatment planning simulations (Wilkens and Oelfke 2004a, 2004b). A strong linear correlation

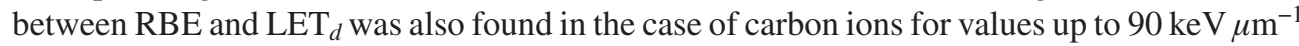
(Ando and Kase 2009).

2.4.1. Proton modelling In this study, a linear model (LM), that assumes a linear relation between $\alpha$ and the $\mathrm{LET}_{d}$, was adopted for protons

$$
\begin{aligned}
& \alpha_{\mathrm{LM}}=\alpha_{0}+m \times \mathrm{LET}_{d} \\
& \beta_{\mathrm{LM}}=\beta
\end{aligned}
$$

where $\alpha_{0}$ is the linear parameter of LQ model in the limit LET $\rightarrow 0$, while $\beta_{\mathrm{LM}}$ is assumed to be independent of LET (Frese et al 2011, Wilkens and Oelfke 2005). $\theta=\left(\alpha_{0}, m, \beta\right)$ represents the 'biological' free parameters of the LM to be adjusted via the likelihood maximization to reproduce the experimental clinical data.

In the present investigation two versions of the LM were considered. In the first approach, denoted as LM1, we kept $\alpha_{0}$ as a free parameter, while setting $m=0$ and $\beta=\beta_{X}$ to the reference radiation value. Neglecting the $\mathrm{LET}_{d}$ dependence in the LM1 implies that a constant RBE is used along the specific organ/tissue. Conversely, in LM2, the $\mathrm{LET}_{d}$ modulation within the treated volume was explicitly accounted for by using $m \neq 0$ as a free parameter to be adjusted via the likelihood maximization and setting $\left(\alpha_{0}, \beta\right)=\left(\alpha_{X}, \beta_{X}\right)$, i.e. to the values of the reference radiation. The latter assumption implies that $\mathrm{RBE} \rightarrow 1$ when $\mathrm{LET}_{d} \rightarrow 0$. Both LM1 and LM2 were used to describe the biological response of the clinical target volume (CTV) and the bladder volumes for which the parameters were adjusted independently. A test was also performed to verify if the parameter $m$ can be considered tissue independent as was proposed in Frese et al (2011). The reference radiation LQ parameters used for the tumor and bladder are summarized in table 2 . These values were derived from the $50 \%$ response dose $D_{50}$, the maximum normalized value of the dose-response gradient $\gamma$ and the ratio $R=\alpha_{X} / \beta_{X}$, as reported in Mavroidis et al (2013).

2.4.2. Carbon ion modelling. Benchmarking of the MKM for carbon ion irradiation showed that the model agrees well with monoenergetic in vitro cell measurements (Kase et al 2008). The model was also used in more complex clinical treatment planning studies (Inaniwa et al 2010) and hence it is another good candidate for the present analysis. A reformulation of the MKM in terms of $\mathrm{LET}_{d}$ was used (named cMKM) for the fitting procedure. According to the cMKM the LQ parameters were calculated as

$$
\begin{aligned}
& \alpha_{\mathrm{cMKM}}=\frac{1-\exp \left(-\left(\alpha_{0}-\beta_{0} \gamma\right) \gamma_{n}\right)}{\gamma_{n}} \\
& \beta_{\mathrm{cMKM}}=\beta
\end{aligned}
$$




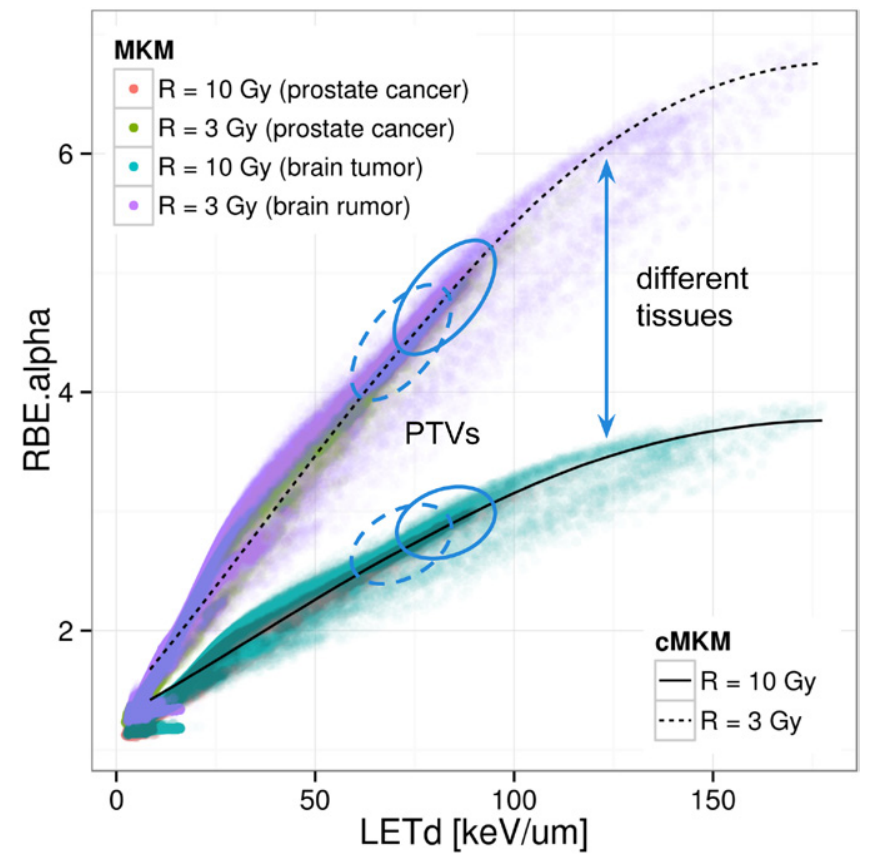

Figure 3. $\mathrm{RBE}_{\alpha}=\alpha / \alpha_{X}$ versus $\mathrm{LET}_{d}$ distribution, calculated at the voxel level for the full body, in two fictitious treatments: a prostate cancer (two opposing irradiation fields) and a pediatric brain tumor case (three coplanar irradiation fields on the coronal plane). Two radiobiological parametrizations were used in both cases corresponding to $\alpha_{X} / \beta_{X}$ ratios $R=3 \mathrm{~Gy}$ and $R=10 \mathrm{~Gy}$. The cloud of points represents the evaluation performed using the MKM and the complete LET spectra for each voxel of the irradiated volumes, while the continuous lines represent the cMKM evaluations. The circled regions represent the loci of the planning target volume (PTV) for the prostate (dashed line circle) and brain tumor (solid line circle) targets.

where $\alpha_{0}$ is the linear parameter of LQ model in the limit LET $\rightarrow 0$, while $\beta$ is assumed LET independent. In this formulation $\beta_{0}$ is an internal parameter of the model and in general $\beta \neq \beta_{0}$. $\gamma=\gamma\left(R_{d}, \mathrm{LET}_{d}\right)$ and $\gamma_{n}=\gamma_{n}\left(R_{n}, \mathrm{LET}_{d}\right)$ are the dose-averaged specific energies delivered in a single event within the domain and cell nucleus, respectively. $R_{d}$ and $R_{n}$ are the radii of the domain and cell nucleus, respectively and, together with $\alpha_{0}, \beta_{0}$ and $\beta$, represent the biological parameters $\theta$ of the cMKM. The averaged specific energies were evaluated using an amorphous track structure model (Kase et al 2008), assuming the irradiation was composed of representative particles with charge $Z=6$ and LET $=\mathrm{LET}_{d}$.

Two versions of the cMKM were considered. In the first one, denoted as cMKM1, we kept $\beta_{0}$ as the only free parameter to be determined via the likelihood maximization, while we set $\alpha_{0}=\alpha_{X}, \beta=\beta_{X}$, equal to the LQ parameters of the reference radiation (see table 2). $R_{n}=3.99 \mu \mathrm{m}$ and $R_{d}=0.37 \mu \mathrm{m}$ were also kept fixed. The latter values correspond to the values used in the reference MKM parametrization. Within this version of the model, the RBE is forced to be 1 in the limit of $\mathrm{LET}_{d} \rightarrow 0$. The choice to use $\beta_{0}$ as the adjustable free parameter was dictated by a sensitive analysis of the model. By changing this parameter it is possible to effectively modulate the steepness of the $\alpha\left(\mathrm{LET}_{d}\right)$ response curve (see for example figure 3 ), the latter having the maximum correlation with the TCP and NTCP estimations and hence with the tissue characterization. In the second version of the model, indicated with cMKM2, 
two free adjustable parameters were considered, namely $\beta_{0}$ and $\beta$, while keeping $\alpha_{0}=\alpha_{X}$, the reference radiation value (table 2 ) and $R_{n}$ and $R_{d}$ equal to the in vitro estimations. Both cMKM1 and cMKM2 were used to describe the biological response of the CTV and the bladder, using independent parametrizations.

2.4.3. $L E T_{d}$ dependence analysis. We verified by comparing the LM and cMKM evaluations with the MKM simulations performed using the complete spectra of the particles, including secondary ions, that the biological effect is mainly dependent on $\mathrm{LET}_{d}$, with little influence on the full LET spectra. It is possible to reproduce the effect predicted by the complete evaluations via a proper choice of the biological parameters $\theta$ (see figure 3 ). We also determined, through simulations of different irradiation conditions, such as variations in beam-setup and patient anatomy, that the biological parametrization of the LM and the cMKM is factorized with respect to the $\mathrm{LET}_{d}$ dependence. This means that, within these models, the biological parametrization of the tissue does not depend on the specific ballistic of the irradiation, but only reflects the biological properties of the tissue (3). The set of biological parameters derived from the fitting procedure on a specific training set can be effectively used for predictive analyses in different irradiation conditions, associated to different $\mathrm{LET}_{d}$ distributions.

2.4.4. RBE evaluation. The LQ parameters evaluated according to the $\mathrm{LM}$ and $\mathrm{cMKM}$ models can be used efficiently to determine RBE values using a formalism that accounts for RBE variation over dose, $\mathrm{LET}_{d}$ and tissue type (Carabe-Fernandez et al 2007, Carabe-Fernandez et al 2010).

$$
\operatorname{RBE}\left(D, \operatorname{LET}_{d}\right)=\frac{R\left(-1+\sqrt{1+4 \beta_{X}\left(\alpha\left(\operatorname{LET}_{d}\right) D+\frac{D^{2}}{R}\right)}\right)}{2 D}
$$

where the $R$ is the reference radiation ratio $\alpha_{X} / \beta_{X}$, while the quadratic parameter $\beta=\beta_{X}$ for the ion irradiation is assumed to be equal to the one of the reference radiation.

\subsection{Clinical response models}

The models used in this study to quantify the TCP/NTCP are based on Poisson distribution assumptions (Munro and Gilbert 1961) taking into account also the tumor characteristic and the organ architecture, respectively (Källman et al 1992)

$$
\pi^{(j)}=\left\{\begin{array}{l}
\operatorname{TCP}^{(j)}=\exp \left[-\mathrm{d} v \sum_{i \in V^{(j)}} n_{i}^{(j)}\right] \\
\mathrm{NTCP}^{(j)}=\left\{1-\prod_{i \in V^{(j)}}\left[1-\exp \left(-n_{i}^{(j)}\right)^{s^{(j)}}\right]^{\mathrm{d} v}\right\}^{1 / s^{(j)}}
\end{array}\right.
$$

where the product is over the voxels $i$ in volume $V^{(j)}$, each of them with sub-volume $\mathrm{d} v, j$ is the index pointing to the specific tissue/organ and $s^{(j)}$ is the relative seriality parameter of the organ in consideration. The expected number of survived clonogens $n_{i}^{(j)}$ was calculated as

$$
n_{i}^{(j)}=n\left(D_{i}, \operatorname{LET}_{\mathrm{d}, i}, N_{f} \mid \theta^{(j)}, \gamma^{(j)}\right)=\exp \left(e \gamma^{(j)}\right) \prod_{f=1}^{N_{f}} p\left(D_{i} / N_{f}, \mathrm{LET}_{\mathrm{d}, i} \mid \theta^{(j)}\right)
$$


where $\gamma^{(j)}$ is a parameter associated with the density of clonogens prior to irradiation, $D_{i}$ is the total dose deposited in the $i$-th voxel, $N_{f}$ is the number of treatment fractions. The $p\left(D_{i} / N_{f}, \mathrm{LET}_{\mathrm{d}, i} \mid \theta^{(j)}\right)$ are the probabilities of the specific endpoint at the cellular level, as defined in equation (1) and are evaluated using the LM (equation (2)) or the cMKM (equation (3)). In this formulation, the clonogen repopulation during treatment is neglected and the full repair between fractions has been also assumed, i.e. the fractions are completely uncorrelated.

The $\theta^{(j)}$ is the set of free biological parameters for the specific tissue/organ, according to the specific radiobiological model, $\theta^{(j)}=\left(\alpha_{0}, m, \beta\right)^{(j)}$ for the LM and $\theta^{(j)}=\left(\alpha_{0}, \beta_{0}, R_{n}, R_{d}, \beta\right)^{(j)}$ for the cMKM. Since the parameters $\gamma$ and $s$ are assumed to be radiation type independent, they were kept fixed to the values reported in literature from x-rays irradiations, $\gamma=4$ for the tumor and $\gamma=3$ and $s=0.18$ for the bladder (Mavroidis et al 2013) (see table 2).

Poisson-based models were used in the present analysis since they are able to evaluate the individual response to the irradiation and to directly use the cell survival probability computed at the voxel level. Population response can be achieved by integrating the response over different individuals (described in the following). Other models that effectively describe a population response have been proposed, e.g. the Marsden model for the TCP (Webb and Nahum 1993) or equivalent uniform dose (Niemierko 1997) EUD-based models, for both TCP (Stavrev et al 2010) and NTCP (Mohan et al 1992, Deasy 2000). However, they need to be recast properly in order to provide the radiobiological coupling we need in our analysis, taking into account the spatially varying radiosensitivity due to the non-uniform LET distribution.

Due to the sparseness of the available data, the simplifying assumption that the biological parameters do not display inter-patient variability was also knowingly made. In other words, the inter-patient variability of the response is assumed to be solely associated to the variability of dose and $\mathrm{LET}_{d}$ distributions among patients and that it does not depend on the variability of the inherent biological parametrization. To account for inter-patient variability of $\theta^{(j)}$, an additional set of parameters $\sigma_{\theta^{(j)}}$ will eventually be introduced in a further development of the model, in which the TCP/NTCP will be integrated, e.g. by means of a Monte Carlo sampling over the parameter distribution, characterized by an average $\theta^{(j)}$ and a standard deviation $\sigma_{\theta^{(j)}}$.

\subsection{Statistical analysis}

The parameters $\theta$ of the LM and cMKM included in the TCP/NTCP models were used as adjustable free parameters to reproduce experimental tissue/organ endpoint (bRFS and TOX data, respectively). Considering the binary nature of the outcome (local recurrence or the occurrence of a complication), the most accurate fitting method is the maximum likelihood estimation and it corresponds to maximize

$L L\left(\theta \mid \operatorname{LET}_{d_{k}}, e_{k}\right)=\sum_{k} e_{k} \cdot \ln \left(\pi_{k}\left(D_{k}, \operatorname{LET}_{d_{k}} \mid \theta\right)\right)+\left(1-e_{k}\right) \cdot \ln \left(1-\pi_{k}\left(D_{k}, \operatorname{LET}_{d_{k}} \mid \theta\right)\right)$

where $L L\left(\theta \mid D_{k}, \mathrm{LET}_{\mathrm{dk}}, e_{k}\right)=L L(\theta)$ indicates the logarithm of the likelihood, $e_{k}$ is the binary outcome ( 0 or 1$)$ for patient $k, \pi_{k}\left(D_{k}, \operatorname{LET}_{\mathrm{d}_{k}} \mid \theta\right)=\pi_{k}(\theta)$ is the estimate of the TCP/NTCP for the parameter set $\theta$ (equation (5)). $D_{k}$ and $\mathrm{LET}_{\mathrm{dk}}$ represent the complete three-dimensional dose and $\mathrm{LET}_{\mathrm{d}}$ distributions found in patient $k$.

Since individual patient data were not available for this study, equation (7) could not be directly applied and a population-based statistics was introduced. Analytical formulations of TCP/NTCP for a population of patients have been proposed in literature to account for the 
heterogeneity of the model parameters and of the dose among the patients (see for example Carlone et al (2006), Schinkel et al (2007), Stavrev et al (2001, 2010) and Webb and Nahum (1993)). In our case the population response was modeled by explicitly evaluating the individual TCP/NTCP over the representative patient set and then averaging the results. The groups were identified based on similarity of treatment with respect to the predictors in the model, corresponding to the treatment setups reported in table 1 . Then the likelihood function to be optimized becomes:

$$
L L(\theta)=\sum_{l=1}^{N} r_{l} \cdot \ln \left(\Pi_{l}(\theta)\right)+\left(n_{l}-r_{l}\right) \cdot \ln \left(1-\Pi_{l}(\theta)\right)
$$

where $n_{l}$ and $r_{l}$ indicate the number of patients and responders (controlled patients/patients with complications) in group $l$, respectively. The summation is performed over the groups of patients, $N=1$ in the case of protons and $N=2$ in the case of carbon ion treatments. $\Pi_{l}(\theta)$ is the average TCP/NTCP over the set of representative patients obtained when the group-specific treatment setup is applied. Thus, for each group

$$
\Pi_{1}(\theta)=\left\langle\pi_{k^{\prime}}^{(l)}\right\rangle=\frac{1}{N_{k^{\prime}}^{(l)}} \sum_{k^{\prime}=1}^{N_{k^{\prime}}^{(l)}} \pi_{k^{\prime}}^{(l)}(\theta)
$$

where $k^{\prime}$ is the representative patient index and $N_{k^{\prime}}^{(l)}=6$ is the number of representative patients in the $l$-th group.

95\% one-dimensional confidence intervals (CI) and correlated confidence regions (CR) were obtained by exploring the value of $L L$ in the neighborhood of its maximum and thus reproducing the entire iso-surfaces defined by

$$
L L_{\mathrm{CI}}=\max (L L)-\frac{1}{2} \chi_{h}^{2}(0.05)
$$

where $h$ is the number of free parameters. Explicitly:

$$
\mathrm{CR}=\left\{\theta \mid L L(\theta) \leqslant L L_{\mathrm{CI}}\right\}
$$

and the corresponding confidence bands $(u, l)$ for a derived quantity, such as the RBE, are defined as

$$
\begin{aligned}
& \operatorname{RBE}_{l}\left(D, \operatorname{LET}_{d}\right)=\left\{\min _{\theta \in \mathrm{CR}} \operatorname{RBE}\left(D, \operatorname{LET}_{d} \mid \theta\right)\right\} \\
& \operatorname{RBE}_{u}\left(D, \operatorname{LET}_{d}\right)=\left\{\max _{\theta \in \mathrm{CR}} \operatorname{RBE}\left(D, \operatorname{LET}_{d} \mid \theta\right)\right\}
\end{aligned}
$$

with $\mathrm{RBE}_{l} \leqslant \mathrm{RBE} \leqslant \mathrm{RBE}_{u}$. Following a principal component analysis approach, a possible linear transformation of the free parameters of the $\mathrm{cMKM} 2,\left(\beta, \beta_{0}\right) \rightarrow\left(\beta, \beta_{1}\right)$, was identified to reduce the strong linear correlation. The transformation is $\beta_{1}=\beta_{0}+k\left(\beta^{*}-\beta\right)$ where $\beta^{*}$ is the value of $\beta$ corresponding to the maximum likelihood and $k$ is an angular coefficient obtained from the eigenvector analysis of the correlation matrix.

The Akaike information criterion (AIC) (Akaike 1974) was used to compare the accuracy of the different models weighting also their complexity in terms of number of free parameters. AIC is mathematically expressed as follows:

$$
\mathrm{AIC}=2 h-2 \max (L L)
$$

The lower the AIC number, the better the model for describing a given dataset. 


\subsection{Degeneration of the solutions}

One of the major limitations of the described method can be found in the possible degeneration of the solutions in the maximization of equation (8). It is likely that, due to the limited amount of available clinical data, it is impossible to properly fix all the parameters of the model. More precisely, treatments with different dose per fraction are required to univocally identify the LQ parameters $\alpha\left(\mathrm{LET}_{d}\right)$ and $\beta$. Furthermore, in order to better discriminate the parameters expressing the $\mathrm{LET}_{d}$ dependence, such as $m$ for the LM and $\beta_{0}$ for the cMKM, different irradiation modalities (beam setups) and dose/fraction are necessary. A general method to resolve this potential degeneration is to modify equation (7) using a Bayesian framework by adding an a-priori likelihood potential $p_{0}(\theta)$ to include the probabilistic information from the in vitro parametrization

$$
L L^{\prime}\left(\theta \mid D_{k}, \operatorname{LET}_{d_{k}}, e_{k}\right)=\ln \left(p\left(D_{k}, \operatorname{LET}_{d_{k}}, e_{k} \mid \theta\right)\right)+\ln \left(p_{0}(\theta)\right)
$$

where the probability distribution $p\left(D_{k}, \mathrm{LET}_{d_{k}}, e_{k} \mid \theta\right)$ is the one used to derive the likelihood in equation (7) and $p_{0}(\theta)$ is obtained from the analysis of in vitro data. To account for the heterogeneous data from in vitro experiments, a bootstrap method can be used to estimate $p_{0}(\theta)$. The Bayesian approach embodied in equation (14) is not included in the present study, but it is introduced here as a natural extension of the implemented method.

\section{Results}

Typical dose and $\mathrm{LET}_{d}$ distributions for proton- and carbon ion-based RT are reported in figure 2, for a single patient. The dose versus $\mathrm{LET}_{d}$ distributions of the complete set of representative patients are reported in figure 4 . The single peak found in the target irradiated with protons is due to the small LET variance and to the usage of a constant RBE $=1.1$ in the inverse planning procedure. The variability and the strong anti-correlation between dose and $\mathrm{LET}_{d}$ observed in the target irradiated with carbon ions, is due to the approximate proportionality $\mathrm{RBE} \propto \mathrm{LET}_{d}$ and the uniform equivalent dose constraint used in the inverse planning. The analyzed cases show the typical spread observed in the dose volume histograms (DVHs) when a patient population is considered. While similar patterns among patients are observed in distributions inside the tumoral target, an irregular behavior is found in the bladder volume, due to the anatomical variance within the population.

\subsection{Prostate treatments with protons}

When considering the proton-based treatments using a single trial (only one fractionation scheme), only one parameter, $\alpha_{0}, m$ or $\beta$, can be determined when the LM is used. The radiobiological parameters $\alpha_{0}$ and $m$ (for LM1 and LM2 respectively) obtained from the likelihood maximization for target and bladder, with their CI, $\max (L L)$ and AIC values are reported in table 3 . In the same table, the values corresponding to the reference planning, in which a constant $\mathrm{RBE}=1.1$ was used, are also reported for comparison. In figure 5, an example of the likelihood profiles obtained in the case of LM1 is displayed. In the same figure the estimates of the bRFS and the G3 toxicity together with their experimental values are also shown.

Exploiting the radiobiological parameters inferred from the clinical data, we re-calculated the RBE distributions in the target and the bladder via equation (4) for the complete patient set. The RBE volume histograms (RVH) of the re-calculated RBE distributions, employing both LM1 and LM2, are pictured in figure 6. In the same figure the results obtained from the 

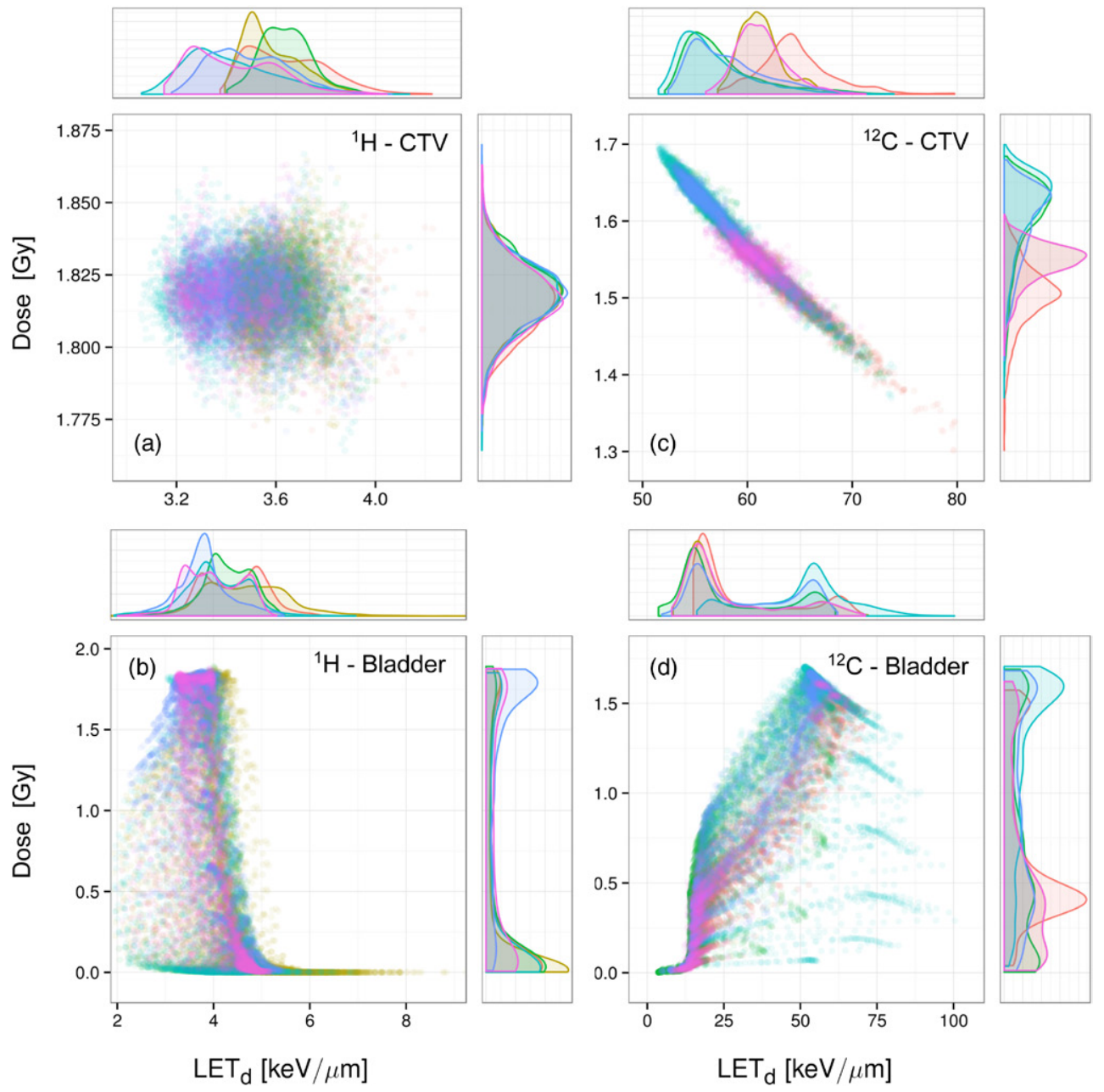

Figure 4. Dose versus $\mathrm{LET}_{d}$ distributions for the full set of representative patients, calculated at the voxel level in the CTV (panels $(a)$ and $(c)$ ) and in the bladder (panels $(b)$ and $(d)$ ), for proton irradiations (panels $(a)$ and $(b)$ ) and carbon ion irradiations (panel $(c)$ and $(d)$. Each dot represents the values of dose and $\mathrm{LET}_{d}$ found in a single voxel belonging to a specific VOI. Marginal distributions are also included in the plots. Different colours are associated with different patients.

reference planning $(\mathrm{RBE}=1.1)$ are also reported for comparison. The results for a specific patient are reported in figure 7, where an estimation of the corresponding CBs via equation (11) is also shown.

An average $\mathrm{RBE}=1.1$ was found, with an $\mathrm{RBE}<1.1$ in the target and $\mathrm{RBE}>1.1$ in the bladder. The modulation of RBE reported in panel $(a)$ and $(b)$ of figure 6 , is due exclusively to its dose dependence (see equation (4)), since in LM1 the $\mathrm{LET}_{d}$ dependence is neglected, $\alpha\left(\mathrm{LET}_{d}\right)=\alpha_{0}$. The spatial gradient of the RBE is larger when using an explicit $\mathrm{LET}_{d}$ dependence for the $\alpha$ parameter, as in LM2, where values up to 1.8 in small volumes of the bladder were found (figure 6, panels $(c)$ and $(d)$ ). 
Table 3. Radiobiological model parameters for the proton-based treatments. $\mathrm{CI}$, AIC and $\max (L L)$ values are also reported. The last two entries of the table correspond to consider the LM2 simultaneously for the CTV and the bladder. Note: the likelihood values were normalized removing a binomial factor common to all models.

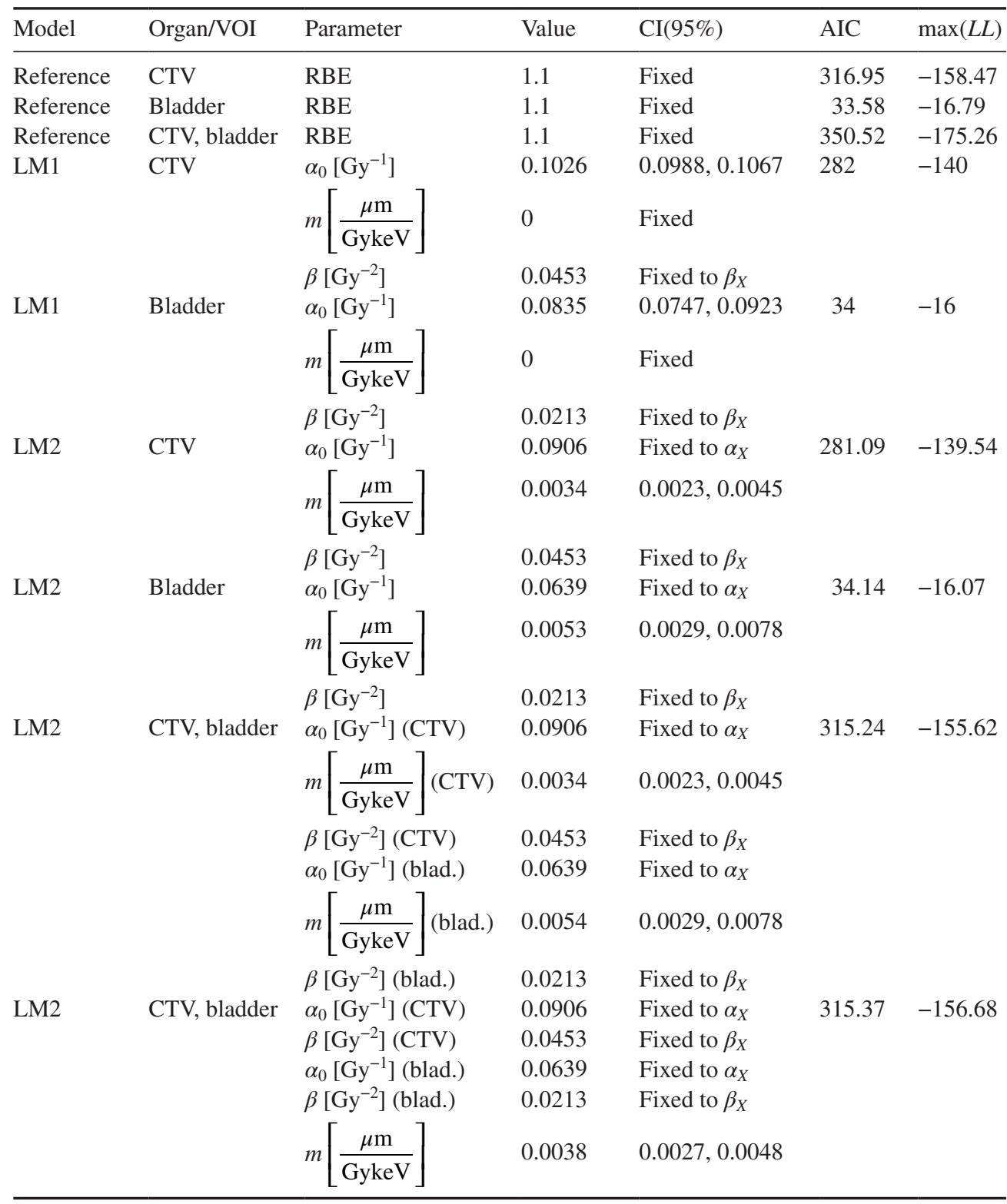

\subsection{Prostate treatments with carbon ions}

In the case of carbon-based RT, two trials were available allowing the determination of up to two parameters of the cMKM. The parameters $\beta_{0}$ for CTV and bladder and the corresponding CI, $\max (L L)$ and AIC values, obtained from the model cMKM1 used to simultaneously fit two 

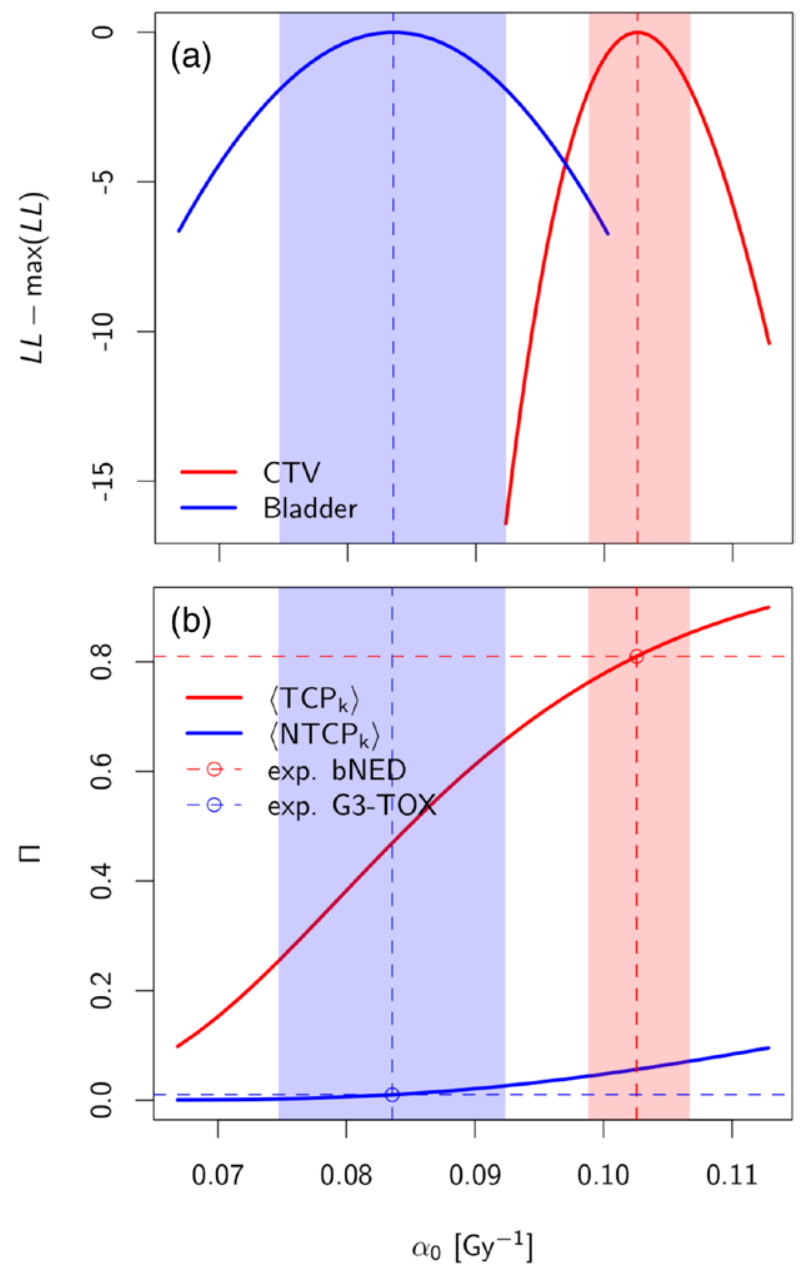

Figure 5. In panel (a), examples of log-likelihood profiles obtained for the $\alpha_{0}$ parameter of LM1 (proton-based treatments), for the CTV and for the bladder, are reported. 95\% $\mathrm{CI}$ are also represented in the plot with shaded areas. In panel $(b)$ the corresponding estimates $\Pi=\left\langle\mathrm{TCP}_{k}\right\rangle$ and $\Pi=\left\langle\mathrm{NTCP}_{k}\right\rangle$, for bRFS and G3 TOX respectively, are also reported together with the experimental values (circles).

different trials, corresponding to $D_{f}=3.15 \mathrm{~Gy}$ and $D_{f}=3.6 \mathrm{~Gy}$, are reported in table 4 . The simultaneous determination of $\beta_{0}$ and $\beta$ was performed using the cMKM2 to concurrently fit the two trials; the corresponding values for CTV and bladder are also reported in the same table. The likelihood landscape for the radiobiological parameters and the corresponding CRs are reported in figure 8 . The radiobiological parameters for the CTV obtained from cMKM1 and cMKM2 are similar, while the AIC value is slightly lower in the case of cMKM1, giving preference to the latter. Negative $\beta$ estimates were obtained for the bladder in the case of the cMKM2, whereas, by construction, the cMKM1 employs positive values of $\beta$. In this case the cMKM1 AIC is slightly higher with respect to cMKM2.

The RBE and equivalent dose for target and bladder have also been re-calculated with the optimized parameter sets, using both the cMKM1 and cMKM2 parametrization. The volume 

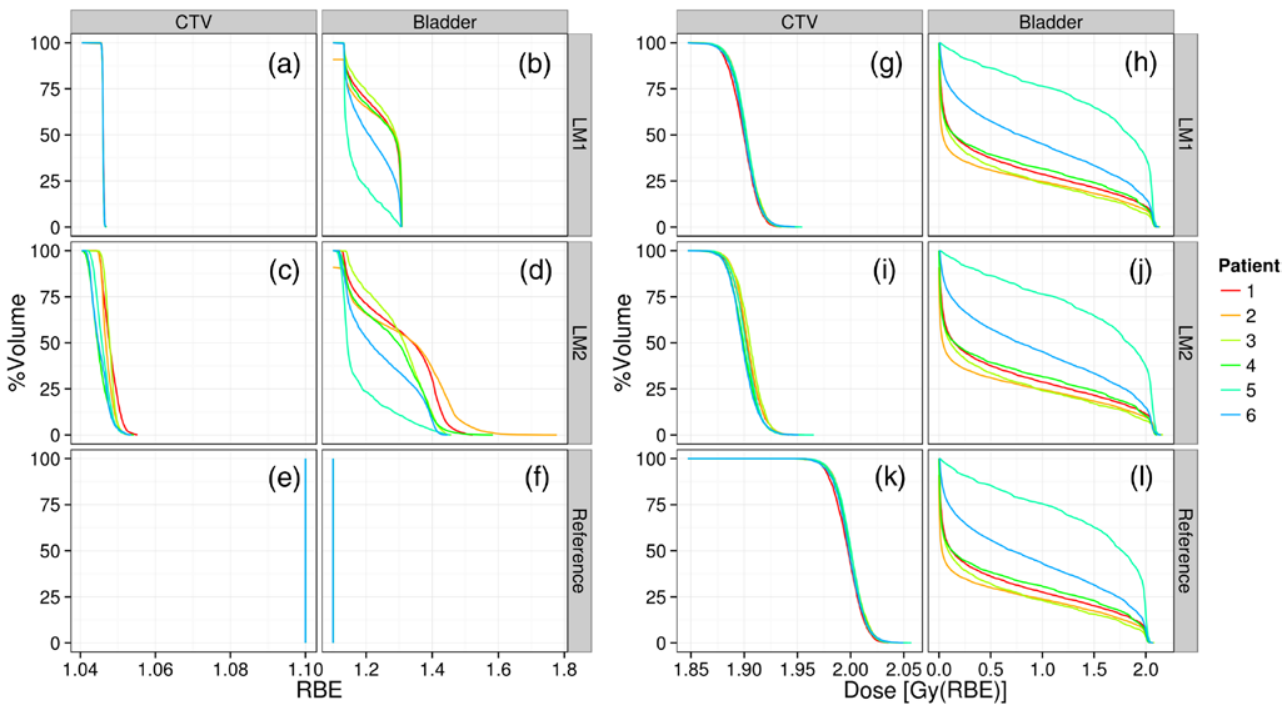

Figure 6. Volume histograms of the RBE and RWD distributions for the proton-based RT trials, evaluated in the CTV and in the bladder for the complete set of representative patients. The evaluations were performed for the reference plan, the LM1 and the LM2 radiobiological parametrizations. In panel $(e)$ and $(f) \mathrm{RBE}=1.1$ for the reference plan is also reported.

histograms of the RBE and equivalent dose distributions evaluated in target and bladder, for the carbon-based RT trial with dose per fraction $D_{f}=3.6 \mathrm{~Gy}(\mathrm{RBE})$, together with those the reference plan, are shown in figure 9 .

\subsection{RBE and RWD volume histogram confidence bands}

The uncertainty associated with the fitted radiobiological parameters translates into an uncertainty in the derived quantities, such as the RBE and the corresponding RWD. The contribution of this specific component to treatment uncertainties was calculated for all the investigated models using equation (12).

In figure 7 the RBE and the RWD volume histograms, evaluated for a single representative patient, are shown for both protons and carbon ions. In the same plot the $95 \%$ confidence bands obtained from the probability distribution of the radiobiological parameters are also reported (shaded area of the same color.)

An average 4\% RBE uncertainty for protons was found in the target RVH, whereas for the bladder a 10\% uncertainty in the low RBE region and 20\% (LM1) and 25\% (LM2) in the high RBE region were found. The same relative uncertainties were also found in the RWD DVHs. Similar results were obtained within the cohort.

In the case of carbon ion-based RT, the RBE uncertainty presents a complex behaviour. For the CTV, average RBE CIs of 2.5\% (cMKM1) and 5\% (cMKM2) were found. For the bladder, average RBE CIs of $8 \%$ (cMKM1) and 15\% (cMKM2) were found. CIs of 2.5\% (cMKM1) and 5\% (cMKM2) for the equivalent dose, for both CTV and bladder, were also found. Similar results were obtained within the cohort. 

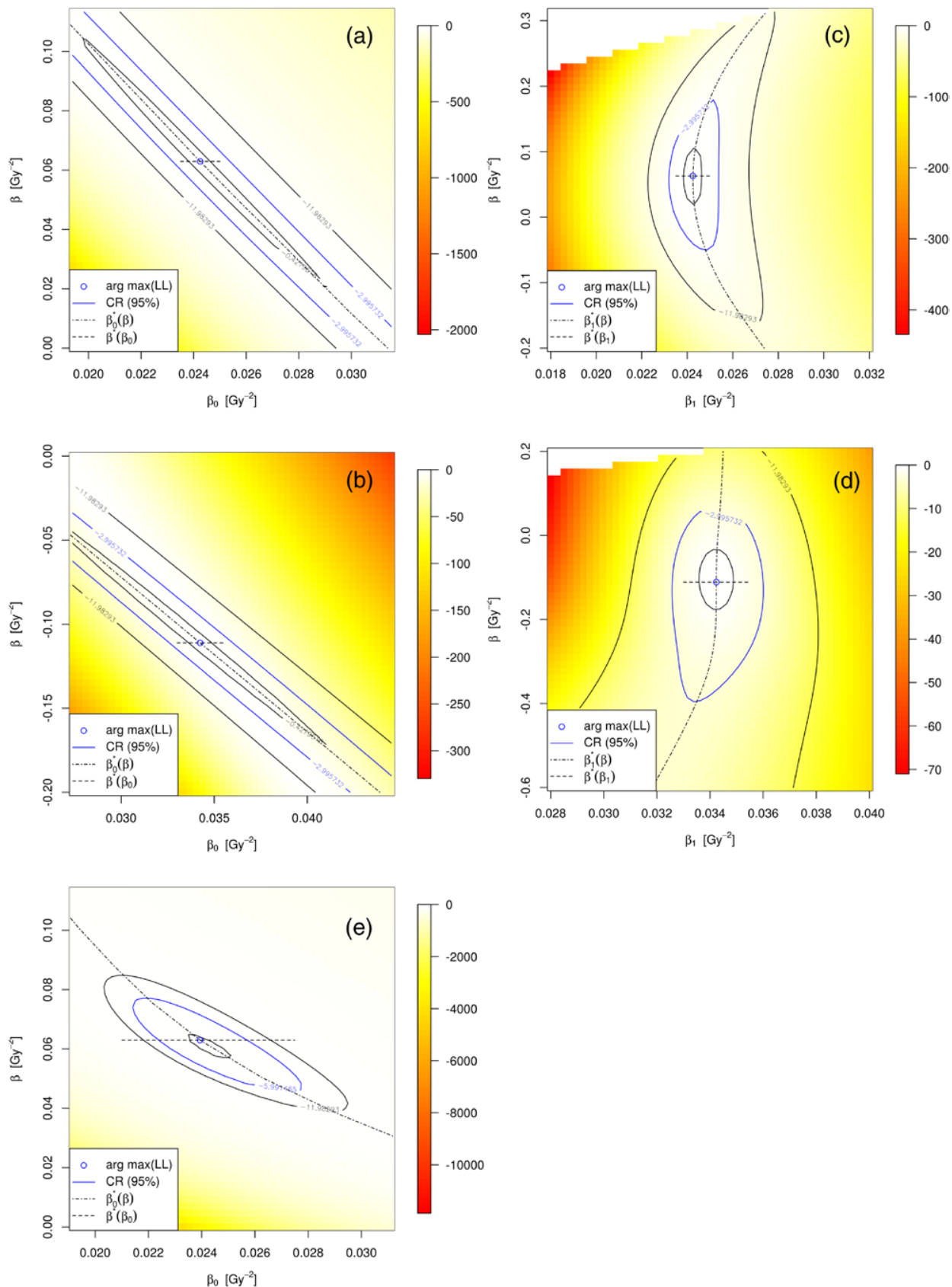

Figure 7. Log-likelihood landscape $(L L-\max (L L))$ obtained from the analysis of two trials with carbon ions $\left(D_{f}=3.15\right.$ and $\left.3.6 \mathrm{~Gy}(\mathrm{RBE})\right)$, using the cMKM2 parametrization. In panels $(a)$ and $(c)$ the CTV log-likelihood landscapes using the original parametrization $\left(\beta_{0}, \beta\right)$ and the transformed one $\left(\beta_{1}, \beta\right)$ are reported. The corresponding bladder loglikelihood landscapes are reported in panel $(b)$ and $(d)$. In panel $(e)$ is reported the CTV evaluation in which a fictitious trial $\left(D_{f}=8 \mathrm{~Gy}(\mathrm{RBE})\right)$ is used together with a real one $\left(D_{f}=3.15 \mathrm{~Gy}(\mathrm{RBE})\right)$. The blue line represents the contour encircling the $95 \% \mathrm{CR}$. The dashed lines represent $L L$ profiles maximized for the complementary parameter: $\beta_{0}^{*}=\arg \max _{\beta}\left(L L\left(\beta_{0}, \beta\right)\right)$ and $\beta^{*}=\arg \max _{\beta_{0}}\left(L L\left(\beta_{0}, \beta\right)\right)$. 

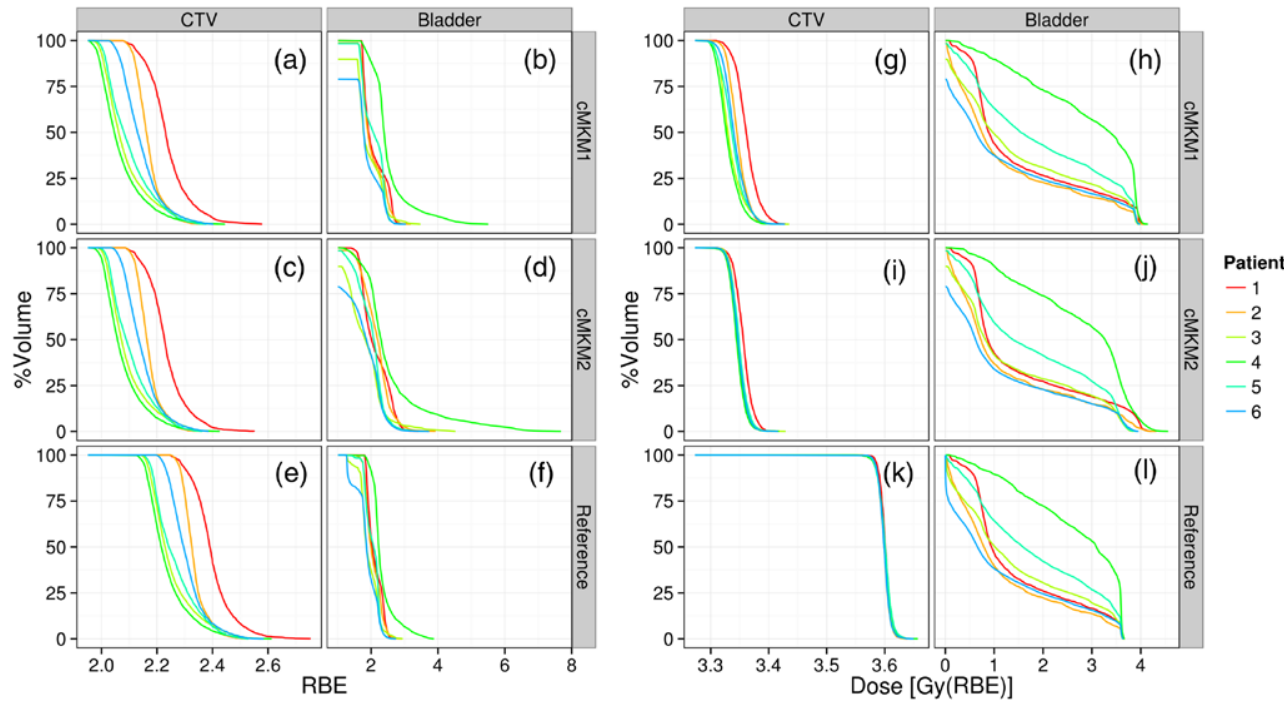

Figure 8. Volume histograms of the RBE and equivalent dose distributions for the carbon-based RT trials, evaluated in the CTV and in the bladder for the complete set of representative patients. The evaluations were performed using the reference plan, the cMKM1 and the cMKM2 radiobiological parametrizations.

Table 4. Radiobiological model parameters for cMKM1 and cMKM2 (carbon ion based treatments). CI, AIC and $\max (L L)$ values are also reported. Note: the likelihood values were normalized removing a binomial factor common to all models.

\begin{tabular}{lllllll}
\hline Model & Organ/VOI & parameter & value & CI $(95 \%)$ & AIC & $\max (L L)$ \\
\hline cMKM1 & CTV & $\alpha_{0}\left[\mathrm{~Gy}^{-1}\right]$ & 0.0906 & Fixed to $\alpha_{X}$ & 281.97 & -139.98 \\
& & $\beta_{0}\left[\mathrm{~Gy}^{-2}\right]$ & 0.0262 & $0.0253,0.0270$ & & \\
& & $\beta\left[\mathrm{Gy}^{-2}\right]$ & 0.0453 & Fixed to $\beta_{X}$ & & \\
cMKM1 & \multirow{2}{*}{ Bladder } & $\alpha_{0}\left[\mathrm{~Gy}^{-1}\right]$ & 0.0639 & Fixed to $\alpha_{X}$ & 147.67 & -72.84 \\
& & $\beta_{0}\left[\mathrm{~Gy}^{-2}\right]$ & 0.0198 & $0.0186,0.0212$ & & \\
& & $\beta\left[\mathrm{Gy}^{-2}\right]$ & 0.0213 & Fixed to $\beta_{X}$ & & \\
cMKM2 & \multirow{2}{*}{ CTV } & $\alpha_{0}\left[\mathrm{~Gy}^{-1}\right]$ & 0.0906 & Fixed to $\alpha_{X}$ & 283.84 & -139.92 \\
& & $\beta_{0}\left[\mathrm{~Gy}^{-2}\right]$ & 0.0242 & Correlated CR & & \\
& & $\beta\left[\mathrm{Gy}^{-2}\right]$ & 0.0629 & Correlated CR & & \\
cMKM2 & \multirow{2}{*}{ Bladder } & $\alpha_{0}\left[\mathrm{~Gy}^{-1}\right]$ & 0.0639 & Fixed to $\alpha_{X}$ & 146.72 & -71.37 \\
& & $\beta_{0}\left[\mathrm{~Gy}^{-2}\right]$ & 0.0342 & Correlated CR & & \\
& & $\beta\left[\mathrm{Gy}^{-2}\right]$ & -0.1111 & Correlated CR & & \\
\hline
\end{tabular}

\section{Discussion}

The implemented procedure allows for an estimation of the radiobiological parameters for target and bladder based on the reported clinical data.

\subsection{RBE and RBE weighted dose distributions}

Regarding protons, an average $\mathrm{RBE}=1.1$ was found, consistently with the current clinical approach. However, in the target the predicted RBE is slightly lower than the assumed 

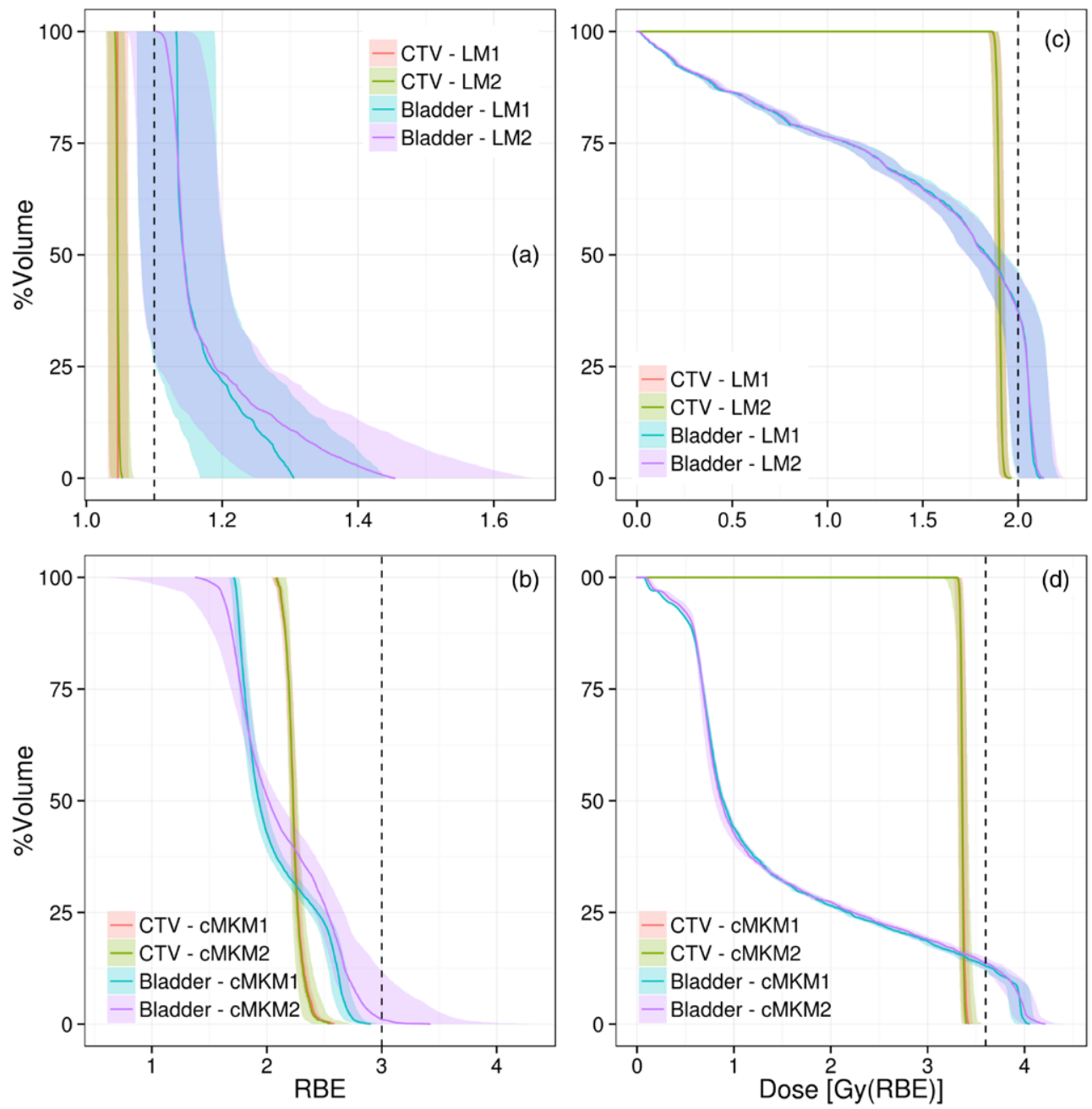

Figure 9. Volume histograms of the RBE and RWD evaluated for a single patient, for proton with models LM1 and LM2 (panels $(a)$ and $(c)$ ) and carbon ion with models cMKM1 and cMKM2 (panels $(b)$ and $(d)$ ). In the same plots the $95 \%$ confidence band obtained from the probability distribution of the radiobiological parameters are reported (shaded area). The dashed lines represents the reference values of the RBE (RBE $=1.1$ for protons and $\mathrm{RBE}=3$ for carbon ions) and of the planned equivalent dose in the CTV ( $D=2 \mathrm{~Gy}(\mathrm{RBE})$ for protons and $D=3.6 \mathrm{~Gy}(\mathrm{RBE})$ for carbon ions).

$\mathrm{RBE}=1.1$; this is in agreement with some analyses and preclinical studies (see e.g. Kagawa et al (2002) and Paganetti et al (2002)). Conversely, the RBE values found in the bladder are larger than 1.1, due to the LET increase at the end of the proton range. Such values are consistent with experimental in vitro observations (Petrović et al 2010, Calugaru et al 2011). This explanation holds also in the case of LM1 where an implicit RBE-LET dependence has $^{2}$ in fact been considered by using different radiobiological parameters for target and bladder. In the case of LM2 analysis, where the RBE-LET ${ }_{d}$ dependence is explicitly accounted for, hot-spots with larger RBE were found in the bladder, as expected from the high LET components due to secondary particles in the border region of the irradiation field (Grassberger 


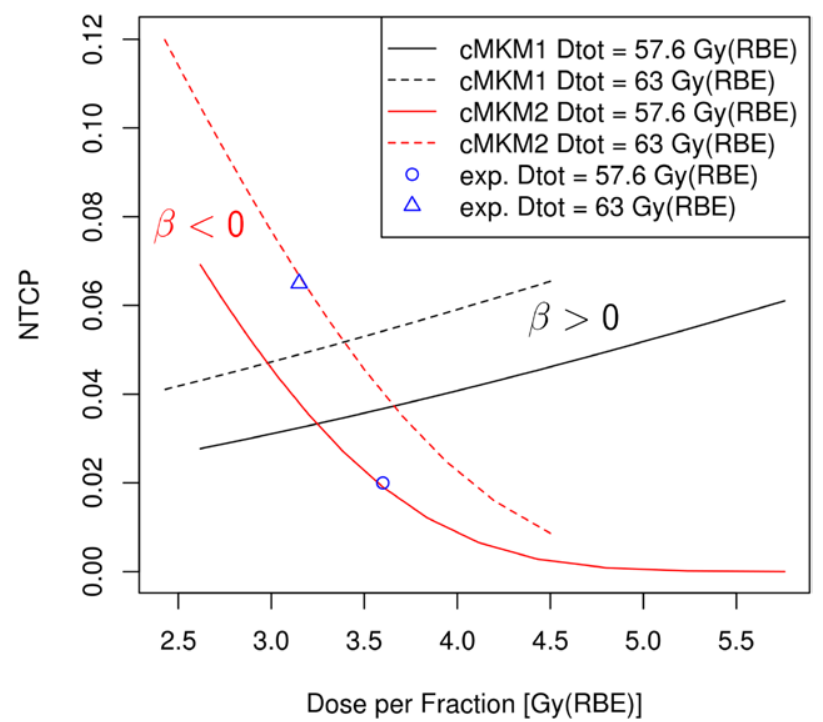

Figure 10. NTCP as a function of dose per fraction (with constant total dose), predicted for carbon ion treatments using cMKM1 and cMKM2. The curves represent treatments with the same total dose. Experimental observations are also reported in the same plot.

and Paganetti 2011), which were considered in the physical model used by the TPS. The AIC values found for the assumption $\mathrm{RBE}=1.1$ are higher than for the variable RBE model (see table 3) in the target, while they were similar for the bladder. Overall, considering both target and bladder, AIC values would support the adoption of a variable RBE in proton TP.

In the case of carbon ion treatments, the RBE and the corresponding RWD in the target, evaluated from the cMKM1/cMKM2 with parameters optimized to reproduce the clinically observed tumor control, are substantially lower than the RBE and RWD used in the reference TP (Okada et al 2012). This indicates that an overestimation of the equivalent dose results from the use of in vitro radiobiological parametrization in the TPS. The situation is inverted in the bladder, where an underestimation from the use of in vitro radiobiological parametrization was observed. Furthermore, a slightly non-uniform RWD in the target was also found in the re-planned treatments, in particular in the case of cMKM1, owing to the different RBE$\mathrm{LET}_{d}$ dependence obtained using the optimized radiobiological parameters with respect to the reference one used in inverse planning. From a modeling point of view, this is due to the

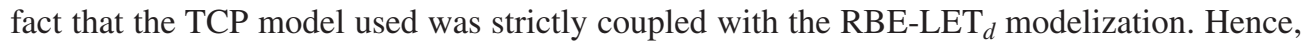
not only the average RBE, but also the specific three-dimensional RBE distribution is directly dependent on the average clinical outcome. This also suggests the risk of a sub-optimal dose distribution in the target and the possibility of increasing the TCP via a proper radiobiological parametrization in the planning phase.

\subsection{Radiobiological parameters}

In the case of proton-based RT the radiobiological parameters obtained for the LM, reported in table 3, show similar trends to those reported in Carabe et al (2012) and Wilkens and Oelfke (2005), where the values were obtained fitting in vitro survival data using similar linear models. However, in some cases the values are different, in particular the parameter $m$, that 
describe the $\mathrm{LET}_{d}$ dependence. The $m$ found in these works $\left(0.01<m<0.02 \mu \mathrm{m} \mathrm{keV}-1 \mathrm{~Gy}^{-1}\right)$ is higher than the ones found with our clinical inference $\left(m=0.0034 \mu \mathrm{m} \mathrm{keV}^{-1} \mathrm{~Gy}^{-1}\right.$ for the CTV and $m=0.0054 \mu \mathrm{m} \mathrm{keV}^{-1} \mathrm{~Gy}^{-1}$ for the bladder). In the case of the LM2 we obtained similar AIC ranking for models that discriminate the parameter $m$ among different tissues (CTV and bladder) and those that use a unique $m$, confirming the approximation used in Frese et al (2011). However, a limited clinical data set was used in our analysis and it is likely that a better discrimination will be feasible as more data is included in the future.

In the case of carbon-based RT, negative $\beta$ values were found in the bladder when applying the cMKM2 model, with the upper extremum of $95 \% \mathrm{CI}$ greater than zero. The obtained results are consistent with the available clinical data (see for example figure 10, where the behavior of the NTCP against the dose per fraction is reported). AIC ranking supports such a result, providing lower values for $\mathrm{cMKM} 2$ with respect to the $\mathrm{cMKM} 1$ (where $\beta=\beta_{X}>0$ ) (see table 4). These values could be due to the heterogeneous cohort included in the two studies, with one population more radiosensitive than the other, as reported in Okada et al (2012). Additional trials could improve the accuracy of predicted $\beta$ value and its CI.

It is worth noting that these estimates, in particular the RBE, are strongly dependent on the choice of the reference radiation parameters. Our choice was derived from literature (Mavroidis et al 2013) and reference therein. Since these parameters have been fitted over a population response, it could be conceptually incorrect to directly use them in an individual model (Schinkel et al 2007, Stavrev et al 2010); however, we assumed that they were not too dissimilar to the putative individual ones in the range of the phenomenological estimates and in the clinically-relevant dose range. Some of these parameters, such as the clonogen density, related to parameter $\gamma$ and the seriality parameter $s$, were also assumed to be radiation independent. However this independence could be an approximation, since the mechanistic reasoning behind the model may be incorrect (Stavreva and Stavrev 2002, AAPM 2012) and these functions are generally used to fit the data phenomenologically. Other parameters, e.g. the ratio $R=\alpha_{X} / \beta_{X}$, play a central role in both the evaluation of the response to $\mathrm{x}$-rays and ion irradiation, the latter in terms of the RBE (Dale and Jones 1999, Carabe-Fernandez et al 2007). In general, these parameters present wide CI, as reported in Bentzen and Ritter (2005), where for the prostate $R \in[-3.3,5.6]$ Gy. Our results suggest a possible general method to determine the radiobiological parameters with smaller CIs for both ion and photon-based treatments, by adding further constraints to x-ray parametrization from the clinical data of ion treatments and vice versa.

\subsection{Degeneration and $\mathrm{Cl}$}

Currently, the applicability of the proposed approach is limited to the low number of available clinical trials and also in terms of low heterogeneity of the adopted fractionation schemes and beam setups (Goitein 2010, Suit et al 2010, Jensen et al 2011). This translates into high CIs and a possible degeneration of the estimated parameters. In fact, the large CIs observed may be reduced by increasing the difference in dose per fraction of the considered trials. In the panel $(e)$ of figure 8 is shown the shrinking of the CR that can be achieved considering two carbon ion-based trials, one clinical, corresponding to a $D_{f}=3.15 \mathrm{~Gy}(\mathrm{RBE})$ and the other fictitious, with $D_{f}=8 \mathrm{~Gy}(\mathrm{RBE})$.

In general, the degeneration and the large $\mathrm{CI}$ issues could be solved by using the Bayesian framework as described above (equation (14)). The Bayesian framework also ensures a more robust evaluation of the radiobiological parameters, by starting from the in vitro experience and then adapting in an incremental manner to the clinical evidence as additional data become available. 
Finally, the possibility to use each plan of the treated patients in the cohort directly, will permit equation (7) to be used, thus avoiding the need to assume a representative patient set and to fit average quantities. This strategy will eventually solve the degeneration problem.

\subsection{Clinical routine aspects}

Remarkably, the proposed procedure does not affect the standard clinical workflow of TP. In other words, it is still possible to use the RBE and the corresponding RWD as reference quantities within TP. However, the models would employ the radiobiological parameters directly inferred from actual clinical outcomes, thereby allowing a more reliable prediction of the clinical response after ion irradiation. In particular, this approach permits the identification of the proper clinical CR of the parameters and the CI of the derived quantities and hence represents a more robust strategy than the commonly used in vitro/in vivo extrapolations for the analysis of the radiobiological uncertainties and their impact in TP (Böhlen et al 2012, Friedrich et al 2013).

As indicated in AAPM (2012), biological models can be used for both plan optimization and evaluation. Both tasks are closely related; however their predictive power may differ depending on whether they are used for plan optimization or for plan evaluation. This approach is an attempt to overcome the generic RBE models commonly used to steer the plan optimization process, towards the implementation of more predictive models to be used for dose-response evaluation and thus to guide the clinical strategy.

\section{Conclusions}

An alternative approach to radiobiological modelling in TP with proton and carbon ion beams has been discussed in this paper. The proposed approach reduces the need to use a reference radiation and/or parameters carried out from in vitro/in vivo studies, since the evaluated absolute biological effect, estimated at the voxel level from TP dose and LET distributions, is directly linked to the clinical outcome. This suggests the possibility of improving the 3D dose distributions in TP using more reliable radiobiological parameters, which can be derived directly from the observed clinical data. Of note, the investigated procedure uses a $\mathrm{LET}_{d}$-based modelization, thus reducing the complexity and overall computational cost required to perform the analysis with respect to approaches that rely on the knowledge of a full LET spectra.

To illustrate the general principle of the proposed approach, we estimated the radiobiological parameters of the $\mathrm{LET}_{d}$-based models, using imaging and contouring from six representative patients, the calculated doses and LETs based on three published schedules for prostate cancer patients and their related clinical outcomes. In the case of protons, an average $\mathrm{RBE}=1.1$ was found, consistently with current usage. However, the re-calculation of the treatment plans using an optimized set of radiobiological parameters obtained for protons and carbon ions clearly showed that the reference plan systematically over- and under-predicts the equivalent dose in the target and bladder, respectively.

The models presented do not account for any inherent inter-patient or intra-organ radiosensitivity variations in their present form, or for tumour cell proliferation or tissue oxygenation, or other clinical factors influencing control and complications, such as concurrent chemotherapy, patients age and preexisting medical conditions. Nevertheless, the proposed method is general and can be extended to include all the above variables.

The major limitation of this method is the lack of available clinical data, in terms of quantity and diversification in schedules and beam setups. However, parameter estimates can be 
easily refined as additional follow-up data become available. A predictive analysis of the model is ongoing in a prospective study on an independent patient population, in order to test the ability of the model to predict the response in new patients.

\section{References}

AAPM Task Group 166TPC 2012 The Use and QA of Biologically Related Models for Treatment Planning Report of AAPM Task Group 166 Med. Phys. 39 1386-409

Akaike H 1974 A new look at the statistical model identification IEEE Trans. Automatic Control $19716-23$

Ando K and Kase Y 2009 Biological characteristics of carbon-ion therapy Int. J. Radiat. Biol. 85 715-28

Bentzen S, Joiner M 2009 Basic Clinical Radiobiology ed M Joiner and A V Kogel (London: Edward Arnold) chapter 9

Bentzen S r M, Constine L S, Deasy J O, Eisbruch A, Jackson A, Marks L B, Ten Haken R K and Yorke E D 2010 Quantitative analyses of normal tissue effects in the clinic (QUANTEC): an introduction to the scientific issues Int. J. Radiat. Oncol. Biol. Phys. 76 S3-9

Bentzen S r M and Ritter M A 2005 The $\alpha / \beta$ ratio for prostate cancer: what is it, really? Radiother. Oncol. $761-3$

Böhlen T T, Brons S, Dosanjh M, Ferrari A, Fossati P, Haberer T, Patera V and Mairani A 2012 Investigating the robustness of ion beam therapy treatment plans to uncertainties in biological treatment parameters Phys. Med. Biol. 57 7983-8004

Calugaru V, Nauraye C, Noël G, Giocanti N, Favaudon V and Mégnin-Chanet F 2011 Radiobiological characterization of two therapeutic proton beams with different initial energy spectra used at the Institut Curie Proton Therapy Center in Orsay Int. J. Radiat. Oncol. Biol. Phys. 81 1136-43

Carabe A, Moteabbed M, Depauw N, Schuemann J and Paganetti H 2012 Range uncertainty in proton therapy due to variable biological effectiveness Phys. Med. Biol. 57 1159-72

Carabe-Fernandez A, Dale R G, Hopewell J W, Jones B and Paganetti H 2010 Fractionation effects in particle radiotherapy: implications for hypo-fractionation regimes Phys. Med. Biol. 55 5685-700

Carabe-Fernandez A, Dale R G and Jones B 2007 The incorporation of the concept of minimum RBE (RbEmin) into the linear-quadratic model and the potential for improved radiobiological analysis of high-LET treatments Int. J. Radiat. Biol. 83 27-39

Carlone M C, Warkentin B, Stavrev P and Fallone B G 2006 Fundamental form of a population TCP model in the limit of large heterogeneity Med. Phys. 331634

Dale R G and Jones B 1999 The assessment of RBE effects using the concept of biologically effective dose Int. J. Radiat. Oncol. Biol. Phys. 43 639-45

Deasy J O, Bentzen S r M, Jackson A, Ten Haken R K, Yorke E D, Constine L S, Sharma A and Marks L B 2010 Improving normal tissue complication probability models: the need to adopt a 'data-pooling' culture Int. J. Radiat. Oncol. Biol. Phys. 76 S151-4

Deasy J O 2000 Comments on the use of the LymanKutcherBurman model to describe tissue response to nonuniform irradiation Int. J. Radiat. Oncol. Biol. Phys. 47 1458-9

Durante M and Loeffler J S 2010 Charged particles in radiation oncology Nat. Rev. Clin. Oncol. 7 37-43

Elsässer T and Scholz M 2007 Cluster effects within the local effect model Radiat. Res. 167 319-29

Fossati P et al 2012 Dose prescription in carbon ion radiotherapy: a planning study to compare NIRS and LEM approaches with a clinically-oriented strategy Phys. Med. Biol. 57 7543-54

Frese M C, Wilkens J J, Huber P E, Jensen A D, Oelfke U and Taheri-Kadkhoda Z 2011 Application of constant versus variable relative biological effectiveness in treatment planning of intensitymodulated proton therapy Int. J. Radiat. Oncol. Biol. Phys. 79 80-8

Friedrich T, Grün R, Scholz U, Elsässer T, Durante M and Scholz M 2013 Sensitivity analysis of the relative biological effectiveness predicted by the local effect model Phys. Med. Biol. 58 6827-49

Friedrich T, Scholz U, Elsässer T, Durante M and Scholz M 2012a Calculation of the biological effects of ion beams based on the microscopic spatial damage distribution pattern Int. J. Radiat. Biol. 88 103-7

Friedrich T, Scholz U, Elsässer T, Durante M and Scholz M 2012b Systematic analysis of RBE and related quantities using a database of cell survival experiments with ion beam irradiation J. Radiat. Res. 54 494-514 
Giantsoudi D, Grassberger C, Craft D, Niemierko A, Trofimov A and Paganetti H 2013 Linear energy transfer-guided optimization in intensity modulated proton therapy: feasibility study and clinical potential Int. J. Radiat. Oncol. Biol. Phys. 87 216-22

Goitein M 2010 Trials and tribulations in charged particle radiotherapy Radiother. Oncol.: J. Eur. Soc. Therapeutic Radiol. Oncol. $9523-31$

Grassberger C and Paganetti H 2011 Elevated LET components in clinical proton beams Phys. Med. Biol. 56 6677-91

Grassberger C, Trofimov A, Lomax A and Paganetti H 2011 Variations in linear energy transfer within clinical proton therapy fields and the potential for biological treatment planning Int. J. Radiat. Oncol. Biol. Phys. 80 1559-66

Grun R, Friedrich T, Kramer M, Zink K, Durante M, Engenhart-Cabillic R and Scholz M 2013 Physical and biological factors determining the effective proton range Med. Phys. $\mathbf{4 0} 111716$

Hawkins R B 1998 A microdosimetric-kinetic theory of the dependence of the RBE for cell death on LET Med. Phys. 25 1157-70

Hawkins R B 2003 A microdosimetric-kinetic model for the effect of non-Poisson distribution of lethal lesions on the variation of RBE with LET Radiat. Res. 160 61-9

IAEA 2008 Relative biological effectiveness in ion beam therapy Technical reports series no. 461 Technical Report 46 (Vienna: International Atomic Energy Agency)

ICRU 2007 Journal of the ICRU (Oxford: Oxford University Press) chapter report 78 pp 21-8

Inaniwa T, Furukawa T, Kase Y, Matsufuji N, Toshito T, Matsumoto Y, Furusawa Y and Noda K 2010 Treatment planning for a scanned carbon beam with a modified microdosimetric kinetic model Phys. Med. Biol. 55 6721-37

Jackson A, Marks L B, Bentzen S r M, Eisbruch A, Yorke E D, Ten Haken R K, Constine L S and Deasy J O 2010 The lessons of QUANTEC: recommendations for reporting and gathering data on dose-volume dependencies of treatment outcome Int. J. Radiat. Oncol. Biol. Phys. 76 S155-60

Jäkel O, Schulz-Ertner D and Debus J 2007 Specifying carbon ion doses for radiotherapy: the Heidelberg approach J. Radiat. Res. 48 87-95

Jensen a D, Münter M W and Debus J 2011 Review of clinical experience with ion beam radiotherapy Br. J. Radiol. 84 S35-47

Jones B, Underwood T S A and Dale R G 2011 The potential impact of relative biological effectiveness uncertainty on charged particle treatment prescriptions Br. J. Radiol. 84 61-9

Kagawa K et al 2002 Preclinical biological assessment of proton and carbon ion beams at hyogo ion beam medical center Int. J. Radiat. Oncol. Biol. Phys. 54 928-38

Källman P, Agren A and Brahme A 1992 Tumour and normal tissue responses to fractionated nonuniform dose delivery Int. J. Radiat. Biol. 62 249-62

Kanai T et al 1999 Biophysical characteristics of HIMAC clinical irradiation system for heavy-ion radiation therapy Int. J. Radiat. Oncol. Biol. Phys. 44 201-10

Kanai T, Furusawa Y, Fukutsu K, Itsukaichi H, Eguchi-Kasai K and Ohara H 1997 Irradiation of mixed beam and design of spread-out Bragg peak for heavy-ion radiotherapy Radiat. Res. 147 78-85

Kase Y, Kanai T, Matsufuji N, Furusawa Y, Elsässer T and Scholz M 2008 Biophysical calculation of cell survival probabilities using amorphous track structure models for heavy-ion irradiation Phys. Med. Biol. 53 37-59

Koike $\mathrm{S}$ et al 2002 Relative biological effectiveness of $290 \mathrm{MeV} \mathrm{u}^{-1}$ carbon ions for the growth delay of a radioresistant murine fibrosarcoma J. Radiat. Res. 43 247-55

Krämer M and Scholz M 2000 Treatment planning for heavy-ion radiotherapy: calculation and optimization of biologically effective dose Phys. Med. Biol. 45 3319-30

Loeffler J S and Durante M 2013 Charged particle therapy-optimization, challenges and future directions Nat. Rev. Clin. Oncol. 10 411-24

Matsufuji N, Kanai T, Kanematsu N, Miyamoto T, Baba M, Kamada T, Kato H, Yamada S, Mizoe J E and Tsujii $\mathrm{H} 2007$ Specification of carbon ion dose at the national institute of radiological sciences (NIRS) J. Radiat. Res. 48 81-6

Mavroidis P, Ferreira B C, Papanikolaou N and Lopes M D C 2013 Analysis of fractionation correction methodologies for multiple phase treatment plans in radiation therapy Med. Phys. 40031715

Mayahara H, Murakami M, Kagawa K, Kawaguchi A, Oda Y, Miyawaki D, Sasaki R, Sugimura K and Hishikawa Y 2007 Acute morbidity of proton therapy for prostate cancer: the hyogo ion beam medical center experience Int. J. Radiat. Oncol. Biol. Phys. $69434-43$

Mohan R, Mageras G S, Baldwin B, Brewster L J, Kutcher G J, Leibel S, Burman C M, Ling C C and Fuks Z 1992 Clinically relevant optimization of 3D conformal treatments Med. Phys. 19933 
Munro T and Gilbert C 1961 The relation between tumour lethal doses and the radiosensitivity of tumour cells Br. J. Radiol. 34 246-51

Niemierko A 1997 Reporting and analyzing dose distributions: a concept of equivalent uniform dose Med. Phys. 24103

Okada T, Tsuji H, Kamada T, Akakura K, Suzuki H, Shimazaki J and Tsujii H 2012 Carbon ion radiotherapy in advanced hypofractionated regimens for prostate cancer: from 20 to 16 fractions Int. J. Radiat. Oncol. Biol. Phys. 84 968-72

Paganetti H, Niemierko A, Ancukiewicz M, Gerweck L E, Goitein M, Loeffler J S and Suit H D 2002 Relative biological effectiveness (RBE) values for proton beam therapy Int. J. Radiat. Oncol. Biol. Phys. 53 407-21

Petrović I, Ristić-Fira A, Todorović D, Korićanac L, Valastro L, Cirrone P and Cuttone G 2010 Response of a radioresistant human melanoma cell line along the proton spread-out Bragg peak Int. J. Radiat. Biol. $86742-51$

Russo G et al 2014 Physical and radiobiological modelling of ion beams in treatment planning through the beamlets superposition approach Phys. Med. Biol. submitted

Schinkel C, Stavreva N, Stavrev P, Carlone M and Fallone B 2007 Functional form comparison between the population and the individual Poisson based TCP models Radiol. Oncol. 41 90-8

Schlampp I et al 2011 Temporal lobe reactions after radiotherapy with carbon ions: incidence and estimation of the relative biological effectiveness by the local effect model Int. J. Radiat. Oncol. Biol. Phys. 80 815-23

Scholz M, Kellerer A M, Kraft-Weyrather W and Kraft G 1997 Computation of cell survival in heavy ion beams for therapy. The model and its approximation Radiat. Environ. Biophys. 36 59-66

Scholz M and Kraft G 1996 Track structure and the calculation of biological effects of heavy charged particles Adv. Space Res. 18 5-14

Sethi R V et al 2014 Patterns of failure after proton therapy in medulloblastoma; linear energy transfer distributions and relative biological effectiveness associations for relapses Int. J. Radiat. Oncol. Biol. Phys. 88 655-63

Stavreva N A and Stavrev P V 2002 Some limitations of the application of the NTCP model describing the response of organs with 'relatively serial' structure Int. J. Radiat. Biol. 78 948-50

Stavrev P, Niemierko A, Stravreva N and Goitein M 2001 The application of biological models to clinical data Physica Medica XVII 2-12

Stavrev P, Schinkel C, Stavreva N, Warkentin B, Carlone M and Fallone B G 2010 Population TCP estimators in case of heterogeneous irradiation: a new discussion of an old problem Acta Oncol. 49 1293-303

Suit $\mathrm{H}$ et al 2010 Proton versus carbon ion beams in the definitive radiation treatment of cancer patients Radiotherapy Oncol. 95 3-22

Tilly N, Johansson J, Isacsson U, Medin J, Blomquist E, Grusell E and Glimelius B 2005 The influence of RBE variations in a clinical proton treatment plan for a hypopharynx cancer Phys. Med. Biol. 50 2765-77

Webb S and Nahum A E 1993 A model for calculating tumour control probability in radiotherapy including the effects of inhomogeneous distributions of dose and clonogenic cell density Phys. Med. Biol. 38 653-66

Wilkens J J and Oelfke U 2004a A phenomenological model for the relative biological effectiveness in therapeutic proton beams Phys. Med. Biol. 49 2811-25

Wilkens J J and Oelfke U 2004b Three-dimensional LET calculations for treatment planning of proton therapy Z. Med. Phys. 14 41-6

Wilkens J J and Oelfke U 2005 Optimization of radiobiological effects in intensity modulated proton therapy Med. Phys. 32 455-65 